\title{
Testing solar lepton mixing sum rules in neutrino oscillation experiments
}

\author{
Peter Ballett, ${ }^{a}$ Stephen F. King, ${ }^{b}$ Christoph Luhn, ${ }^{c}$ Silvia Pascoli ${ }^{a}$ and \\ Michael A. Schmidt ${ }^{d}$ \\ ${ }^{a}$ IPPP, Department of Physics, Durham University, South Road, Durham DH1 3LE, U.K. \\ ${ }^{b}$ Physics and Astronomy, University of Southampton, Southampton, SO17 1BJ, U.K. \\ ${ }^{c}$ Theoretische Physik 1, Naturwissenschaftlich-Technische Fakultät, Universität Siegen, \\ Walter-Flex-Straße 3, 57068 Siegen, Germany \\ ${ }^{d}$ ARC Centre of Excellence for Particle Physics at the Terascale, \\ School of Physics, The University of Sydney, NSW 2006, Australia \\ E-mail: peter.ballett@durham.ac.uk, king@soton.ac.uk, \\ christoph.luhn@uni-siegen.de, silvia.pascoli@durham.ac.uk, \\ m.schmidt@physics.usyd.edu.au
}

AbStract: Small discrete family symmetries such as $\mathrm{S}_{4}, \mathrm{~A}_{4}$ or $\mathrm{A}_{5}$ may lead to simple leading-order predictions for the neutrino mixing matrix such as the bimaximal, tribimaximal or golden ratio mixing patterns, which may be brought into agreement with experimental data with the help of corrections from the charged-lepton sector. Such scenarios generally lead to relations among the parameters of the physical leptonic mixing matrix known as solar lepton mixing sum rules. In this article, we present a simple derivation of such solar sum rules, valid for arbitrary neutrino and charged lepton mixing angles and phases, assuming only $\theta_{13}^{\nu}=\theta_{13}^{e}=0$. We discuss four leading-order neutrino mixing matrices with $\theta_{13}^{\nu}=0$ which are well motivated from family symmetry considerations. We then perform a phenomenological analysis of the scope to test the resulting four solar sum rules, highlighting the complementarity between next-generation neutrino oscillation experiments such as the reactor experiment JUNO and a superbeam experiment.

Keywords: Neutrino Physics, Discrete and Finite Symmetries

ARXIV EPRINT: 1410.7573 


\section{Contents}

1 Introduction 1

2 Mixing sum rules from charged-lepton corrections 5

2.1 A simple derivation 5

2.2 Fully specified mixing patterns 8

$\begin{array}{lll}2.3 & \text { Common partially constrained patterns } & 10\end{array}$

3 Numerical results $\quad 10$

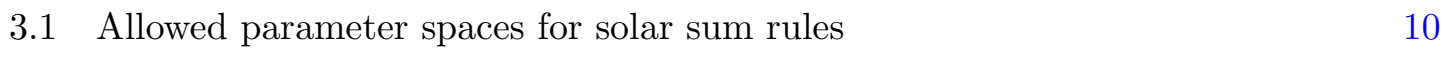

$\begin{array}{lll}3.2 & \text { Simulation details } & 13\end{array}$

$\begin{array}{lll}3.2 .1 & \text { JUNO } & 13\end{array}$

$\begin{array}{lll}3.2 .2 & \text { Wide-band superbeam } & 14\end{array}$

$\begin{array}{lll}3.3 & \text { Simulation results } & 15\end{array}$

4 Beyond $\theta_{13}^{\nu}=0 \quad 17$

$\begin{array}{llr}5 & \text { Renormalisation group corrections } & 19\end{array}$

$\begin{array}{llr}6 & \text { Conclusion } & 20\end{array}$

$\begin{array}{ll}\text { A Simple approximation to the mixing sum rule } & 21\end{array}$

B Neutrino mixing patterns from $\mathbb{Z}_{2} \times \mathbb{Z}_{2}$

\section{Introduction}

Following the measurement of the third lepton mixing angle, the so-called reactor angle $\theta_{13} \approx 8.5^{\circ}[1,2]$, neutrino physics has entered the precision era. Indeed all three lepton mixing angles are expected to be measured with increasing precision over the coming years, with forthcoming accurate measurements expected for both the atmospheric angle $\theta_{23}$ and the solar angle $\theta_{12}$. First hints of the CP-violating (CPV) phase $\delta$ have also been reported in global fits [3-5], and rapid progress can be expected with the next generation of oscillation experiments.

The measurement of the reactor angle has had a major impact on models of neutrino mass and mixing, ruling out at a stroke models based on tribimaximal (TBM) lepton mixing [6], although, as we shall discuss in this paper, these patterns may survive in the neutrino sector, if charged-lepton corrections are included. Such TBM patterns can arise from "direct" models [7], in which the full Klein symmetry ( $S, U$ generators) of the neutrino mass matrix as well as the $T$ symmetry of the charged lepton mass matrix are 
subgroups of an underlying discrete family symmetry. Alternatively, TBM mixing can arise from "indirect" models based on constrained sequential dominance (CSD) [8] with special family symmetry breaking vacuum alignments.

In response to the experimental data, many different model building directions capable of accounting for the reactor angle have emerged, as recently reviewed in refs. [9] and [10]. The viability of these ideas can only be established by comparison with experiment, and a tractable approach to test large classes of models is to identify generic types of prediction associated with these models. A promising example of such a signature can be found in lepton mixing sum rules, which relate the three lepton mixing angles to the CPV oscillation phase $\delta$, or more precisely to $\cos \delta$. Indeed, given the precisely measured values of the mixing angles, they can be regarded as predictions for $\cos \delta$, to be tested in future experiments. Lepton mixing sum rules arise from two distinct types of scenarios and lead to two different types, referred to as atmospheric and solar sum rules $[9,10]$.

Atmospheric sum rules [11] arise from a variety of "semi-direct" models in which only half of the Klein symmetry emerges from the discrete family symmetry, classified in terms of finite von Dyck groups, with charged lepton mixing controlled by the $T$ generator [12-14]. For example, such models can lead to trimaximal-1 (TM1) or trimaximal-2 (TM2) mixing, in which the first or second column of the TBM mixing matrix is preserved, eq. (1.1) and eq. (1.2) respectively,

$$
\begin{array}{ll}
\text { TM1 : } & \left|U_{e 1}\right|=\sqrt{\frac{2}{3}} \quad \text { and } \quad\left|U_{\mu 1}\right|=\left|U_{\tau 1}\right|=\frac{1}{\sqrt{6}} ; \\
\text { TM2 : } & \left|U_{e 2}\right|=\left|U_{\mu 2}\right|=\left|U_{\tau 2}\right|=\frac{1}{\sqrt{3}} .
\end{array}
$$

The atmospheric sum rule $a=\lambda r \cos \delta+\mathcal{O}\left(a^{2}, r^{2}\right)$ can be derived from these conditions [11], where $a \equiv \sqrt{2} \sin \theta_{23}-1, r \equiv \sqrt{2} \sin \theta_{13}$ and $\lambda=1$ for TM1 and $\lambda=-1 / 2$ for TM2. The study of correlations of this type, and their application to the discrimination between underlying models, has been shown to be a realistic aim for a next-generation superbeam experiment [14].

It was first shown in refs. [15] and [16] that $\mathrm{A}_{4}$ generally leads to a "semi-direct model" which predicts TM2 mixing with the second atmospheric sum rule, while the indirect CSD2 model with special family symmetry breaking vacuum alignments $(0,1,1)^{T}$ and $(1,2,0)^{T}$ in ref. [17] predicts TM1 mixing and the first atmospheric sum rule. In fact the TM1 atmospheric sum rule arises from all generalised versions of $\operatorname{CSD}(n)$, based on the vacuum alignments $(0,1,1)^{T}$ and $(1, n, n-2)^{T}$ for integer $n \geq 1[18,19]$, since such alignments are orthogonal to the first column of the TBM matrix, $(2,-1,1)^{T} / \sqrt{6}$, and hence predict TM1 mixing.

The Pontecorvo-Maki-Nakagawa-Sakata (PMNS) matrix $U$ can be expressed as the product of the diagonalising matrices of the neutrino and charged lepton mass terms, $U_{\nu}$ and $U_{e}$, respectively,

$$
U=U_{e}^{\dagger} U_{\nu}
$$

Solar sum rules $[8,20,21]$ arise in models in which a leading-order mixing matrix $U_{\nu}$ is corrected by a small basis change from the charged leptons. In the first models of this 
type $U_{e}$ had a Cabibbo-like form: if we denote the angles which parameterise $U_{\alpha}$ by $\theta_{i j}^{\alpha}$, the angles obey $0 \approx \theta_{13}^{e} \approx \theta_{23}^{e} \ll \theta_{12}^{e} \approx \theta_{C}$. These scenarios are motivated by Grand Unified Theories (GUTs) where the approximately diagonal charged lepton mass matrix is related to the down-type quark mass matrix, together with the assumption that quark mixing arises predominantly from the down-type quark sector. Indeed this was the case in the CSD model where solar sum rules were first proposed [8]. In the context of "direct" models, solar sum rules arise when the full Klein group continues to emerge from the discrete family symmetry (leading for example to TBM mixing in the neutrino sector) while the $T$ generator which governs the charged leptons is broken. In the simple case where only $\theta_{12}^{e}, \theta_{12}^{\nu}, \theta_{23}^{\nu}$ are non-zero, with $\theta_{23}^{e}=\theta_{13}^{e}=\theta_{13}^{\nu}=0$, the charged-lepton corrections do not change the third row of the neutrino mixing matrix, and solar sum rules can be derived from the conditions [22]

$$
\left|U_{\tau 1}\right|=s_{12}^{\nu} s_{23}^{\nu}, \quad\left|U_{\tau 2}\right|=c_{12}^{\nu} s_{23}^{\nu}, \quad\left|U_{\tau 3}\right|=c_{23}^{\nu},
$$

where $s_{i j}^{\alpha} \equiv \sin \theta_{i j}^{\alpha}$ and $c_{i j}^{\alpha} \equiv \cos \theta_{i j}^{\alpha}$. For example, with TBM neutrino mixing $s_{23}^{\nu}=c_{23}^{\nu}=$ $1 / \sqrt{2}, s_{12}^{\nu}=1 / \sqrt{3}$ and $c_{12}^{\nu}=\sqrt{2 / 3}$

$$
\left|U_{\tau 1}\right|=\frac{1}{\sqrt{6}}, \quad\left|U_{\tau 2}\right|=\frac{1}{\sqrt{3}}, \quad\left|U_{\tau 3}\right|=\frac{1}{\sqrt{2}} .
$$

The solar sum rule $s=r \cos \delta+\mathcal{O}\left(a^{2}, r^{2}, s^{2}\right)$ can be derived from these conditions [11], where $s \equiv \sqrt{3} \sin \theta_{12}-1$. It is clear that the conditions on $\left|U_{\tau 1}\right|$ and $\left|U_{\tau 2}\right|$ in eq. (1.4) are identical to the corresponding conditions for TM1 and TM2 mixing, respectively. However the conditions on the other elements of the PMNS mixing matrix are different, so the resulting atmospheric and solar sum rules will also be different.

In this paper, we extend the above derivation of the solar sum rule to the more general case where not only $\theta_{12}^{e}, \theta_{12}^{\nu}, \theta_{23}^{\nu}$ are non-zero but also $\theta_{23}^{e}$ is allowed to be non-zero and all complex phases are kept arbitrary, while still keeping $\theta_{13}^{e}=\theta_{13}^{\nu}=0$. As a result we shall find the remarkable condition,

$$
\frac{\left|U_{\tau 1}\right|}{\left|U_{\tau 2}\right|}=\frac{s_{12}^{\nu}}{c_{12}^{\nu}}=t_{12}^{\nu} .
$$

Of course, the condition in eq. (1.5) can be trivially derived from eq. (1.3), assuming $\theta_{23}^{e}=0$. The notable feature is that eq. (1.5) also holds independently of $\theta_{23}^{e}$ and of all complex phases. However, eq. (1.3) involves further relations which only hold for $\theta_{23}^{e}=0$. These further relations can be used to eliminate the atmospheric angle, providing a solar sum rule which is more restrictive than that coming from eq. (1.5) alone. Nevertheless, we shall continue to refer to the relation in eq. (1.5) as a solar sum rule, since it is satisfied even when $\theta_{23}^{e}=0$, as in eq. (1.3), and is distinct from the atmospheric sum rules discussed earlier. The solar sum rule in eq. (1.5) may be cast as a prediction for $\cos \delta$, as a function of the measured mixing angles and $\theta_{12}^{\nu}$,

$$
\cos \delta=\frac{t_{23} s_{12}^{2}+s_{13}^{2} c_{12}^{2} / t_{23}-s_{12}^{\nu 2}\left(t_{23}+s_{13}^{2} / t_{23}\right)}{\sin 2 \theta_{12} s_{13}},
$$

an expression which had been derived previously using an alternative argument in refs. [23] and [24]. We also highlight a second remarkable feature of eq. (1.6), namely that it is not 
only independent of $\theta_{23}^{e}$ but also of $\theta_{23}^{\nu}$. The sum rule in eq. (1.6) is specified by only fixing the value of $s_{12}^{\nu}$. Therefore, we can enumerate the viable models of this type by deriving the values of $\theta_{12}^{\nu}$ associated with those leading-order mixing patterns with $\theta_{13}^{\nu}=0$ which are derivable from considerations of symmetry. In this article, we shall show that this leads us to four well-motivated solar sum rules: one based on TBM mixing [6] where $s_{12}^{\nu}=1 / \sqrt{3}$, one based on bimaximal (BM) mixing [25-27] where $s_{12}^{\nu}=1 / \sqrt{2}$ and two patterns based on versions of golden ratio mixing including GR1 with $t_{12}^{\nu}=1 / \varphi$ [28-30] and GR3 with $c_{12}^{\nu}=\varphi / \sqrt{3}[31,32]$, where $\varphi=\frac{1+\sqrt{5}}{2}$ is the golden ratio. We shall also discuss the viability of two leading-order patterns which have been invoked in the literature called GR2 with $\theta_{12}^{\nu}=\pi / 5$ [33, 34], and hexagonal (HEX) mixing with $\theta_{12}^{\nu}=\pi / 6$ [35].

For each viable prediction we perform a study of the scope to test the sum rule in eq. (1.6) within the current experimental programme. Over the next few decades, significant new information will be provided on the leptonic mixing matrix from two main sources: the next generation of medium-baseline reactor (MR) experiments and long-baseline wideband superbeams (WBB). The MR programme primarily seeks to measure the interference between atmospheric and solar neutrino oscillations at baseline distances of around 50-60 km. These facilities have been shown to be sensitive to the mass hierarchy [36-39]. There are two main experiments working towards a MR facility, both building on successful measurements of $\theta_{13}$ at a shorter baseline: JUNO [40] and RENO-50 [41, 42]. The WBB experiments can be seen as complementary to the MR proposals. Collaborations such as LBNE [43] and LBNO [44] intend to construct a high-power long-baseline neutrino and antineutrino beam which can exploit matter effects and large statistics for the primary aim of constraining the CPV phase $\delta$. The combination of MR and WBB facilities will provide new levels of precision in the neutrino sector, with $\theta_{12}$ and $\theta_{13}$ being probed to the level of percent by MR experiments, and $\delta$ being constrained by dedicated WBB facilties. This complementarity offers for the first time the possibility of experimentally testing relations such as eq. (1.6). ${ }^{1}$ In this work, we shall simulate illustrative MR and WBB facilities with an aim to exploring how their complementarity can be used to constrain the models of charged-lepton corrections.

The idea of correcting a leading-order neutrino mixing pattern by contributions from the charged leptons has recently been revisited [23, 24, 46-48]. Our work goes beyond these analyses in three ways. Firstly, we present a novel derivation of the correlation in eq. (1.6) in a more general setting, showing it to be the consequence of the simpler relation eq. (1.5), which helps to highlight its relationship to the earlier solar sum rules. Secondly, we systematically derive the possible leading-order mixing patterns from considerations of residual symmetry, finding a small well-motivated set. Thirdly, we present the results of simulations assessing the potential to constrain the solar sum rules from two upcoming complementary oscillation experiments: a superbeam and a reactor facility.

The layout of the remainder of the paper is as follows: in section 2, we present a simple derivation of the correlations induced by charged-lepton corrections. We then sys-

\footnotetext{
${ }^{1}$ For another application of this complementarity to the study of flavour-symmetric predictions, see ref. [45].
} 
tematically identify the viable leading-order neutrino mixing matrices, and comment on their relation to the underlying flavour symmetry. Section 3 is devoted to our numerical study. We first consider the currently allowed parameter spaces of these correlations, then we present the details and results of our simulations of a superbeam and reactor experiment, showing how these can be used to test these relations. We comment on the case where $\theta_{13}^{\nu} \neq 0$ in section 4 , and discuss renormalisation group effects in section 5 . Finally, section 6 concludes the paper.

\section{Mixing sum rules from charged-lepton corrections}

In the first subsection, we present a simple derivation of the solar sum rule of eq. (1.6). Then in later subsections we discuss the leading-order mixing patterns which one encounters in the considered class of models. We shall find that there are only four well-motivated patterns of interest, whose relation to model building will be discussed.

\subsection{A simple derivation}

In the equations that follow, superscripts are attached to quantities which are naturally associated with the neutrinos or the charged leptons (e.g. $\theta^{\nu}$ and $\theta^{e}$ ), whilst physical parameters go without.

Assuming $^{2} \theta_{13}^{\nu}=\theta_{13}^{e}=0$, the PMNS matrix is given by the product of five unitary matrices

$$
U=U_{12}^{e \dagger} U_{23}^{e \dagger} R_{23}^{\nu} R_{12}^{\nu} P^{\nu}
$$

the three right-most matrices describe the neutrino sector, and are parameterised by

$$
R_{23}^{\nu}=\left(\begin{array}{ccc}
1 & 0 & 0 \\
0 & c_{23}^{\nu} & s_{23}^{\nu} \\
0 & -s_{23}^{\nu} & c_{23}^{\nu}
\end{array}\right) \quad \text { and } \quad R_{12}^{\nu}=\left(\begin{array}{ccc}
c_{12}^{\nu} & s_{12}^{\nu} & 0 \\
-s_{12}^{\nu} & c_{12}^{\nu} & 0 \\
0 & 0 & 1
\end{array}\right)
$$

and $P^{\nu}$ is a diagonal matrix of uni-modular complex numbers. The two unitary matrices on the left of eq. (2.1) characterise the charged-lepton corrections, and will be allowed to include extra complex phases,

$$
\begin{aligned}
U_{23}^{e} & =\left(\begin{array}{ccc}
1 & 0 & 0 \\
0 & c_{23}^{e} & s_{23}^{e} e^{-i \delta_{23}^{e}} \\
0-s_{23}^{e} e^{i \delta_{23}^{e}} & c_{23}^{e}
\end{array}\right), \\
U_{12}^{e} & =\left(\begin{array}{ccc}
c_{12}^{e} & s_{12}^{e} e^{-i \delta_{12}^{e}} & 0 \\
-s_{12}^{e} e^{e} \delta_{12}^{e} & c_{12}^{e} & 0 \\
0 & 0 & 1
\end{array}\right) .
\end{aligned}
$$

With these definitions, it is simple enough to compute the explicit form of the PMNS matrix. However, our derivation focuses only on the first two elements of the bottom row

\footnotetext{
${ }^{2}$ It is possible to derive sum rules with $\theta_{13}^{\nu} \neq 0$. We comment on one example in section 4 .
} 
of the physical PMNS matrix, which are found to be

$$
\begin{aligned}
& U_{\tau 1}=s_{12}^{\nu}\left(s_{23}^{\nu} c_{23}^{e}-c_{23}^{\nu} s_{23}^{e} e^{i \delta_{23}^{e}}\right), \\
& U_{\tau 2}=-c_{12}^{\nu}\left(s_{23}^{\nu} c_{23}^{e}-c_{23}^{\nu} s_{23}^{e} e^{i \delta_{23}^{e}}\right) .
\end{aligned}
$$

By comparing eq. (2.2) to the PDG parameterisation of $U$ [49], we find the relations between the physical parameters and our internal parameters,

$$
\begin{aligned}
& \left|U_{\tau 1}\right|=\left|s_{23} s_{12}-s_{13} c_{23} c_{12} e^{i \delta}\right|=\left|s_{12}^{\nu}\left(s_{23}^{\nu} c_{23}^{e}-c_{23}^{\nu} s_{23}^{e} e^{i \delta_{23}^{e}}\right)\right|, \\
& \left|U_{\tau 2}\right|=\left|s_{23} c_{12}+s_{13} c_{23} s_{12} e^{i \delta}\right|=\left|c_{12}^{\nu}\left(s_{23}^{\nu} c_{23}^{e}-c_{23}^{\nu} s_{23}^{e} e^{i \delta_{23}^{e}}\right)\right| .
\end{aligned}
$$

As the ratio of these two equations is independent of the values of the parameters in $U_{23}^{e}$ and $U_{12}^{e}$, we are left with a correlation between observable parameters and the value of the neutrino mixing parameter $\theta_{12}^{\nu}$,

$$
\frac{\left|U_{\tau 1}\right|}{\left|U_{\tau 2}\right|}=\frac{\left|s_{23} s_{12}-s_{13} c_{23} c_{12} e^{i \delta}\right|}{\left|s_{23} c_{12}+s_{13} c_{23} s_{12} e^{i \delta}\right|}=t_{12}^{\nu}
$$

This correlation will be referred to as the solar mixing sum rule. It can be viewed as a predictive statement about the physical CPV phase: squaring both sides of eq. (2.3) and solving for $\cos \delta$ leads us to the expression in eq. (1.6), which we repeat below,

$$
\cos \delta=\frac{t_{23} s_{12}^{2}+s_{13}^{2} c_{12}^{2} / t_{23}-s_{12}^{\nu 2}\left(t_{23}+s_{13}^{2} / t_{23}\right)}{\sin 2 \theta_{12} s_{13}} .
$$

An equivalent correlation has been derived previously using a lengthier argument in refs. [23] and [24]. Understanding its application to specific models, its compatibility with global data and its potential use as a signature of new physics will be the focus of the rest of this article.

The correlation in eq. (1.6) is in fact the full non-linear version of a more familiar firstorder relation. We collect a number of phenomenologically interesting approximations in appendix A. If we expand eq. (1.6) in a small parameter $\varepsilon$, assumed to control the deviation from a leading-order neutrino mixing pattern with maximal atmospheric mixing,

$$
\theta_{13} \sim\left|\theta_{12}-\theta_{12}^{\nu}\right| \sim\left|\theta_{23}-\frac{\pi}{4}\right| \sim \varepsilon
$$

we find the well-known first-order relation $[8,20,21]$,

$$
\theta_{12}=\theta_{12}^{\nu}+\theta_{13} \cos \delta+\mathcal{O}\left(\varepsilon^{2}\right)
$$

The validity of this approximation is dependent upon the severity of the assumptions in eq. (2.5). This can only be assessed on a model dependent basis; however, in figure 1 we show the size of the error $\Delta(\cos \delta) \equiv \cos \delta_{\text {linear }}-\cos \delta$ which is introduced by the linear approximation for the patterns which we will derive in subsections 2.2 and 2.3. Apart from the patterns denoted GR3 and BM (which we will argue in the following section are strongly disfavoured by current data), the error approximately satisfies $|\Delta(\cos \delta)| \lesssim 0.1$. 

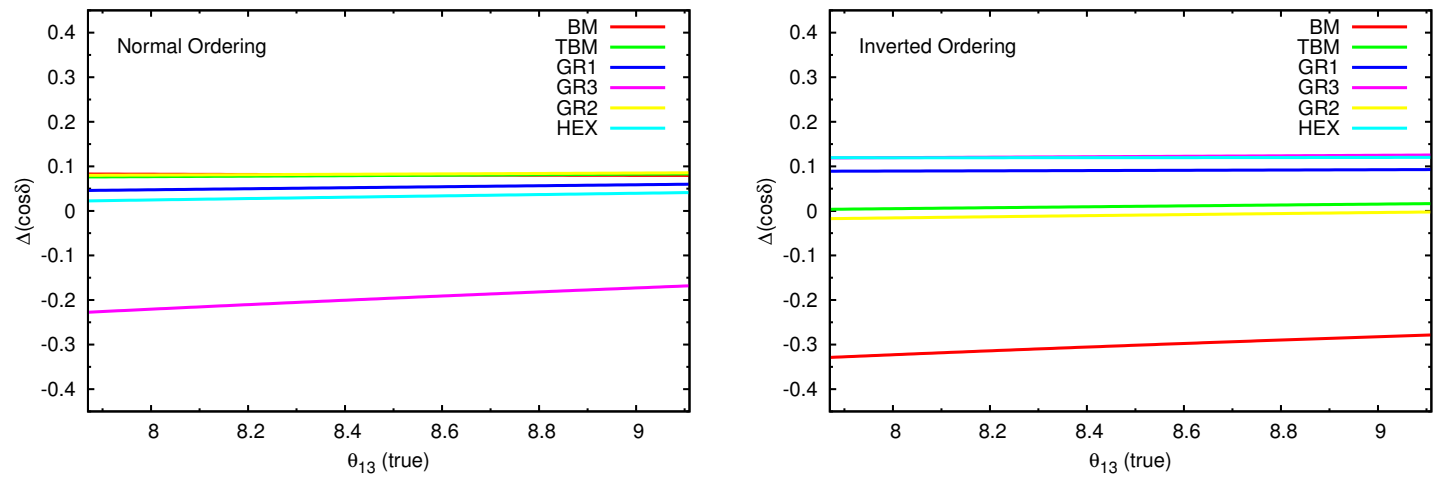

Figure 1. The difference between the linearised expression $\cos \delta_{\text {linear }} \equiv\left(\theta_{12}-\theta_{12}^{\nu}\right) / \theta_{13}$ and the solar sum rule in eq. (1.6). These plots assume $\theta_{12}=33.5^{\circ}$ and take $\theta_{23}$ to be the best-fit value for normal (inverted) ordering in the left (right) panel. The best-fit values are those of ref. [3].

As deviations of this size are expected to be close to the attainable precision at a nextgeneration oscillation facility, all subsequent numerical work will use the full correlations in eq. (1.6). ${ }^{3}$

The solar sum rule derived above is valid for any neutrino mixing pattern with $\theta_{13}^{\nu}=0$ and for any charged-lepton corrections with $\theta_{13}^{e}=0$. Our focus in this work is on the predictions of models which apply charged-lepton corrections to neutrino mixing matrices which are completely fixed by symmetry. In recent work, significant progress has been made in the categorisation of fully-specified mixing patterns subject to some weak model building assumptions. In subsection 2.2, we shall identify a set of leading-order predictions with $\theta_{13}^{\nu}=0$ from arguments of symmetry by following two categorisation schemes from the literature $[12,52]$. As we shall explain, strictly speaking, one of these frameworks [12] is a subcase of the other [52]; however, its systematic exploration has not been presented before, and we shall show how this more restrictive scenario still finds all of the cases of the more comprehensive analysis, while shedding light on the group structure of the viable solutions.

In subsection 2.3, we shall also comment on some mixing patterns frequently invoked in the literature which are not found in the systematic derivations. We will discuss these patterns in the context of an infinite family of neutrino mixing matrices which are partially constrained by symmetry. For the lack of a symmetric origin, we believe these patterns to be more poorly motivated; however, we shall include them in our numerical analysis for completeness.

\footnotetext{
${ }^{3}$ It has been argued [24, 48] that the ratio of leading-order to exact predictions indicate that the linearized sum rules are not accurate enough for phenomenological use. We believe that for many purposes the linearized expressions would be adequate: constant errors of $\Delta(\cos \delta)=0.1$ induce an error of less than $15^{\circ}\left(10^{\circ}\right)$ for $76 \%(60 \%)$ of the range of $\delta$. Therefore the linearized expressions well describe the correlation to the precision of the first phases of the next-generation of superbeams, which expect a sensitivity of $15-30^{\circ}[50,51]$; however, the full expressions will be necessary in the subsequent phases, where precisions are expected to be $8-18^{\circ}[50,51]$.
} 


\section{$2.2 \quad$ Fully specified mixing patterns}

An impressively comprehensive account of fully specified leading-order mixing matrices has been presented in ref. [52]. In this work, it was assumed that neutrinos are Majorana particles, that a finite flavour group $G$ is broken into the Klein group $G_{\nu}=\mathbb{Z}_{2} \times \mathbb{Z}_{2}$ in the neutrino sector, and $G_{e}=\mathbb{Z}_{n}$, with $n \in \mathbb{N}$, in the charged-lepton sector. Such an arrangement completely specifies the leading-order mixing matrix. Under these general assumptions, it was shown that the only possible mixing matrices are given by 17 sporadic patterns and one infinite family of patterns (up to row and column permutations). In ref. [52], all 17 sporadic patterns are shown to be excluded by the current global neutrino oscillation data at $3 \sigma$, whilst the infinite family is allowed for some values of its parameters. In the current work, we are expecting corrections to the leading-order mixing angles of a magnitude $\theta_{13}$, and therefore we have rather more lenient criteria for viability. We define the eligible leading-order mixing patterns as those which meet the criteria $\theta_{13}^{\nu} \leq 20^{\circ}, 20^{\circ} \leq \theta_{12}^{\nu} \leq 45^{\circ}$ and $30^{\circ} \leq \theta_{23}^{\nu} \leq 60^{\circ}$. Scanning over the patterns found in ref. [52] (including row and column permutations), we find that the 17 sporadic patterns allow 13 viable matrices. The infinite family meets our criteria for about $20 \%$ of its allowed parameter space. However, our present aim is to discuss situations where a leading-order pattern with $\theta_{13}^{\nu}=0$ can be brought in-line with observation through corrections from the charged-lepton sector. If we therefore restrict our attention to patterns with $\theta_{13}^{\nu}=0$, we find only 4 patterns which pass our lax phenomenological conditions on the remaining two neutrino mixing angles $\theta_{12}^{\nu}$ and $\theta_{23}^{\nu}$. It is interesting to note that all 4 patterns differ from one another only in their value for the solar mixing angle $\theta_{12}^{\nu}$ with the atmospheric mixing angle fixed at $\theta_{23}^{\nu}=45^{\circ}$.

The first eligible pattern is known as bimaximal (BM) mixing. It has a maximal solar mixing angle [25-27], and is given by a matrix of the form

$$
U_{\mathrm{BM}}^{\nu}=\left(\begin{array}{ccc}
\frac{1}{\sqrt{2}} & \frac{1}{\sqrt{2}} & 0 \\
-\frac{1}{2} & \frac{1}{2} & \frac{1}{\sqrt{2}} \\
\frac{1}{2} & -\frac{1}{2} & \frac{1}{\sqrt{2}}
\end{array}\right)
$$

The second pattern is the tribimaximal (TBM) mixing matrix. This has been associated with models based on the flavour symmetries $\mathrm{A}_{4}$ and $\mathrm{S}_{4}$. It predicts a solar mixing angle given by $s_{12}^{\nu}=1 / \sqrt{3}$, i.e. $\theta_{12}^{\nu} \approx 35.3^{\circ}$. The mixing matrix is given explicitly by

$$
U_{\mathrm{TBM}}^{\nu}=\left(\begin{array}{ccc}
\sqrt{\frac{2}{3}} & \frac{1}{\sqrt{3}} & 0 \\
-\frac{1}{\sqrt{6}} & \frac{1}{\sqrt{3}} & \frac{1}{\sqrt{2}} \\
\frac{1}{\sqrt{6}} & -\frac{1}{\sqrt{3}} & \frac{1}{\sqrt{2}}
\end{array}\right) .
$$

The remaining two patterns both associate the golden ratio $\varphi=\frac{1+\sqrt{5}}{2}$ with the solar mixing angle, although in different ways. The first is the original golden ratio mixing pattern (GR1) [28-30], related to the flavour symmetry $A_{5}$. It predicts $t_{12}^{\nu}=1 / \varphi$, i.e. 
$\theta_{12}^{\nu} \approx 31.7^{\circ}$, resulting in the mixing matrix

$$
U_{\mathrm{GR} 1}^{\nu}=\left(\begin{array}{ccc}
\frac{\varphi}{\sqrt{2+\varphi}} & \frac{1}{\sqrt{2+\varphi}} & 0 \\
-\frac{1}{\sqrt{4+2 \varphi}} & \frac{\varphi}{\sqrt{4+2 \varphi}} & \frac{1}{\sqrt{2}} \\
\frac{1}{\sqrt{4+2 \varphi}} & -\frac{\varphi}{\sqrt{4+2 \varphi}} & \frac{1}{\sqrt{2}}
\end{array}\right) .
$$

The other golden ratio pattern found by our survey is less well known but has been found previously in refs. [31] and [32]. It is associated with the group $A_{5}$ breaking into a $\mathbb{Z}_{3}$ symmetry in the charged-lepton sector and the Klein symmetry in the neutrino sector. This pattern $(\mathrm{GR} 3)$ predicts $c_{12}^{\nu}=\varphi / \sqrt{3}$, i.e. $\theta_{12}^{\nu} \approx 20.9^{\circ}$, leading to a mixing matrix of the form

$$
U_{\mathrm{GR} 3}^{\nu}=\left(\begin{array}{ccc}
\frac{\varphi}{\sqrt{3}} & \frac{\varphi_{g}}{\sqrt{3}} & 0 \\
-\frac{\varphi_{g}}{\sqrt{6}} & \frac{\varphi}{\sqrt{6}} & \frac{1}{\sqrt{2}} \\
\frac{\varphi_{g}}{\sqrt{6}} & -\frac{\varphi}{\sqrt{6}} & \frac{1}{\sqrt{2}}
\end{array}\right),
$$

where $\varphi_{g}$ is the Galois $\left(\mathbb{Q}\right.$-) conjugate of $\varphi$ given by $\varphi_{g}=\frac{1-\sqrt{5}}{2}$. To make the connection with the nomenclature of Fonseca and Grimus [52]: BM is known as $\mathcal{C}_{1}$, TBM is the only member of the infinite family $\mathcal{C}_{2}$ with $\theta_{13}^{\nu}=0$, GR1 is $\mathcal{C}_{11}$ and GR3 is known as $\mathcal{C}_{12}$.

We will now show that these four patterns can also be derived under the framework of refs. $[12,13]$ in which the assumption of $\theta_{13}^{\nu}=0$ will be shown to be unnecessary. This scenario can be seen as a subcase of the previous systematic analysis, also working under the assumption of Majorana neutrinos and a finite group $G$ broken into distinct residual symmetries amongst the charged-lepton sector and the neutrino sector. However, a further assumption is made on the form of the finite groups: they are assumed to be overgroups of the von Dyck groups $[12,13]$,

$$
D(2, m, p)=\left\langle S, T, W \mid S^{2}=T^{m}=W^{p}=S T W=1\right\rangle .
$$

Finiteness of $D(2, m, p)$ restricts the values of $\{m, p\}$ to either $\{3,3\},\{3,4\},\{3,5\}$ or $\{2, N\}$, where the first three choices are associated with the groups $A_{4}, S_{4}$ and $A_{5}$, respectively, while the fourth is related to the dihedral groups $D_{2 N}$. The fully specified mixing matrices were not systematically derived in ref. [13], and we will now sketch this calculation, deferring details to appendix B. It is particularly interesting to note that the further constraint on the form of the group in this framework makes the restriction $\theta_{13}^{\nu}=0$ unnecessary: the only patterns meeting our phenomenological selection criteria are the four previous patterns with $\theta_{13}^{\nu}=0$.

Taking the generators of the symmetry of the charged lepton mass terms to be $T$ and those of the Klein group acting on the neutrino mass terms as $S_{1}$ and $S_{2}$, constraints can be derived on the leading-order mixing matrix directly. Disregarding cases related to the dihedral symmetries, ${ }^{4}$ we find that the patterns are specified by a choice of two parameters $\eta_{1}$ and $\eta_{2}$ taken from the set

$$
\left\{\frac{1}{3}, \frac{2}{3}, \frac{1}{2}, \frac{1+\varphi}{3}, \frac{2-\varphi}{3}, \frac{2+\varphi}{5}, \frac{3-\varphi}{5}\right\}
$$

\footnotetext{
${ }^{4}$ We comment on the option where $\{m, p\}=\{2, N\}$ at the end of appendix B.
} 
subject to the unitarity constraint $\eta_{1}+\eta_{2} \leq 1$. The squared moduli of the elements of the mixing matrix are then given by the following pattern (up to row and column permutations)

$$
\left|U_{\alpha i}^{\nu}\right|^{2}=\left(\begin{array}{ccc}
\eta_{1} & \eta_{2} & 1-\eta_{1}-\eta_{2} \\
\frac{1-\eta_{1}}{2} & \frac{1-\eta_{2}}{2} & \frac{\eta_{1}+\eta_{2}}{2} \\
\frac{1-\eta_{1}}{2} & \frac{1-\eta_{2}}{2} & \frac{\eta_{1}+\eta_{2}}{2}
\end{array}\right) .
$$

Of all the possible combinations of $\eta_{1}$ and $\eta_{2}$, only four leading-order neutrino mixing patterns are eligible by our criteria on $\theta_{i j}^{\nu}$ stated at the beginning of this subsection. They are exactly those found in our discussion above: bimaximal mixing, tribimaximal mixing, and two patterns associated with the golden ratio (GR1 and GR3).

\subsection{Common partially constrained patterns}

There are a few other common mixing patterns with $\theta_{13}^{\nu}=0$. However, these patterns are not found in the systematic surveys of the previous subsection. We will focus on two patterns of this type mentioned in the literature: one associated with the golden ratio (GR2) [33, 34] and one called hexagonal mixing (HEX) [35]. Both patterns have maximal atmospheric mixing $\theta_{23}^{\nu}=\pi / 4$ and vanishing reactor angle $\theta_{13}^{\nu}=0$, but they differ in their predictions for $\theta_{12}^{\nu}$,

$$
\theta_{12}^{\nu}=\frac{\pi}{5} \quad(\mathrm{GR} 2) \quad \text { and } \quad \theta_{12}^{\nu}=\frac{\pi}{6} \quad(\mathrm{HEX})
$$

These predictions can be understood as part of a family of patterns which predict $\theta_{12}^{\nu}=\frac{\pi d}{N}$ with $d, N \in \mathbb{N}$ and $0<d<N$. These are commonly connected to the dihedral groups $D_{2 N}$, and indeed it is possible to derive partial constraints consistent with these patterns by breaking $D_{2 N}$ to different preserved subgroups in the charged-lepton sector and the neutrino sector [53]. Such a construction can generate the prediction

$$
\cos \theta_{12}^{\nu} \cos \theta_{13}^{\nu}=\cos \left(\frac{\pi d}{N}\right),
$$

which leads to the patterns of interest if we fix $\theta_{13}^{\nu}=0$ by hand. Furthermore, the assumption of a dihedral group as the fundamental flavour symmetry does not permit the unification of the three families into a single irreducible representation of the symmetry group. ${ }^{5}$ For these reasons we consider these mixing patterns to be on less firm footing than the models arising from the construction presented previously. Despite these reservations, we will include the GR2 and HEX neutrino mixing patterns in our later analysis. However, we would like to point out that any rational multiple of $\pi$ can be found for $\theta_{12}^{\nu}$ using a suitably large dihedral group in this fashion; GR2 and HEX are only distinguished in that they are the best-fitting predictions of the form $\theta_{12}^{\nu}=\frac{\pi}{N}$.

\section{Numerical results}

\subsection{Allowed parameter spaces for solar sum rules}

The parameter correlations discussed above can be seen as predictions for the remaining unknown parameter $\delta$. We will require $\cos \delta$ to lie in the physical region, for which $-1 \leq$

\footnotetext{
${ }^{5}$ All dihedral groups have irreducible representations of dimensions 1 and 2 only.
} 
$\cos \delta \leq 1$. This may not occur for all models considered or in all the allowed parameter space. In this section, we will consider the predictions of the solar sum rules over the current $3 \sigma$ interval of the mixing angles. We will use the values from v2 of the NuFit collaboration [3]. This parameter space is denoted by $I_{3 \sigma}$,

$$
\begin{aligned}
I_{3 \sigma} & =I_{12} \times I_{13} \times I_{23} \\
& =\left[31.29^{\circ}, 35.91^{\circ}\right] \times\left[7.87^{\circ}, 9.11^{\circ}\right] \times\left[38.3^{\circ}, 53.3^{\circ}\right] .
\end{aligned}
$$

Models which apply $\theta_{12}^{e}$ and $\theta_{23}^{e}$ charged-lepton corrections to a neutrino mixing matrix defined by $\theta_{12}^{\nu}$ and $\theta_{23}^{\nu}$ result in a single constraint given by eq. (1.6). For a given choice of $\theta_{12}^{\nu}$ this formula may only predict physical values for $\cos \delta$ in a subregion of the allowed interval $I_{3 \sigma}$. In figure 2 we show the regions in which the sum rule makes a consistent prediction for $\cos \delta$ (coloured bands) for six different models. In all panels, $\theta_{13}$ is given by the abscissa, $\theta_{12}$ is denoted by the different coloured bands whose width is generated by varying $\theta_{23}$ over its range in $I_{3 \sigma}$. For bimaximal mixing $(\mathrm{BM})$ in the neutrino sector $\left(\theta_{12}^{\nu}=45^{\circ}\right)$ the only regions of parameter space for which we find a consistent prediction require a large value of $\theta_{13}$, a large negative value of $\cos \delta$ and a large value of $\theta_{12}$. This is easily understood from the linearised relation shown in eq. (2.6), where the leading-order prediction of $\theta_{12}^{\nu}=45^{\circ}$ must receive large negative corrections to be brought in agreement with the global data. For tribimaximal mixing (TBM) in the neutrino sector $\left(\theta_{12}^{\nu}=35.3^{\circ}\right)$, smaller values of $\theta_{13}$ are allowed, and all points in $I_{3 \sigma}$ lead to consistent predictions. The predicted values of $\cos \delta$ show only a slight dependence on the true value of $\theta_{13}$ and $\theta_{23}$, lying between $-0.7 \lesssim \cos \delta \lesssim 0.2$. We consider two models referred to as golden ratio mixing: GR1 and GR3. For GR1, all values of $\theta_{12}, \theta_{13}$ and $\theta_{23}$ in $I_{3 \sigma}$ allow for a consistent definition of $\cos \delta$, whereas for GR3 we require a small value of $\theta_{12}$, a large value of $\theta_{13}$ and large positive $\cos \delta$. In figure 2 , we see that GR1 predicts mostly positive values of $\cos \delta$ with $0 \lesssim \cos \delta \lesssim 0.7$. The small region of parameter space in which GR3 is consistent with the data is analogous to the allowed regions of the BM pattern; however, the predictions of $\cos \delta$ for these two models are distinct. The bottom row in figure 2 shows the possible predictions for the two patterns related to dihedral symmetries: GR2 and HEX, which are associated with $\mathrm{D}_{10}$ and $\mathrm{D}_{12}$, respectively. These models make similar predictions to TBM and GR1, and they give physical values of $\cos \delta$ over the whole range $I_{3 \sigma}$. Our results, shown in figure 2, are in agreement with an independent survey of charged-lepton corrections presented for the cases of TBM, GR1, GR2 and HEX in ref. [48].

So far we have considered all points in $I_{3 \sigma}$ on equal footing. However, the corners of this parameter space are arguably less likely: they require large deviations from the current best-fits in multiple parameters. To fully account for this effect we would need a measure of the degree of correlation amongst the parameters inferred from global fits. In figure 3, in the absence of this information, we show the posterior probability density functions for $\cos \delta$ assuming that the likelihood functions for the squared sines of the mixing angles are given by independent Gaussian distributions centred on the current best-fit values and with the widths of the global minima. We take a flat prior in $\sin ^{2} \theta_{i j}$, although we have checked that flat priors in $\theta_{i j}$ do not significantly change the result. This helps to see the most 

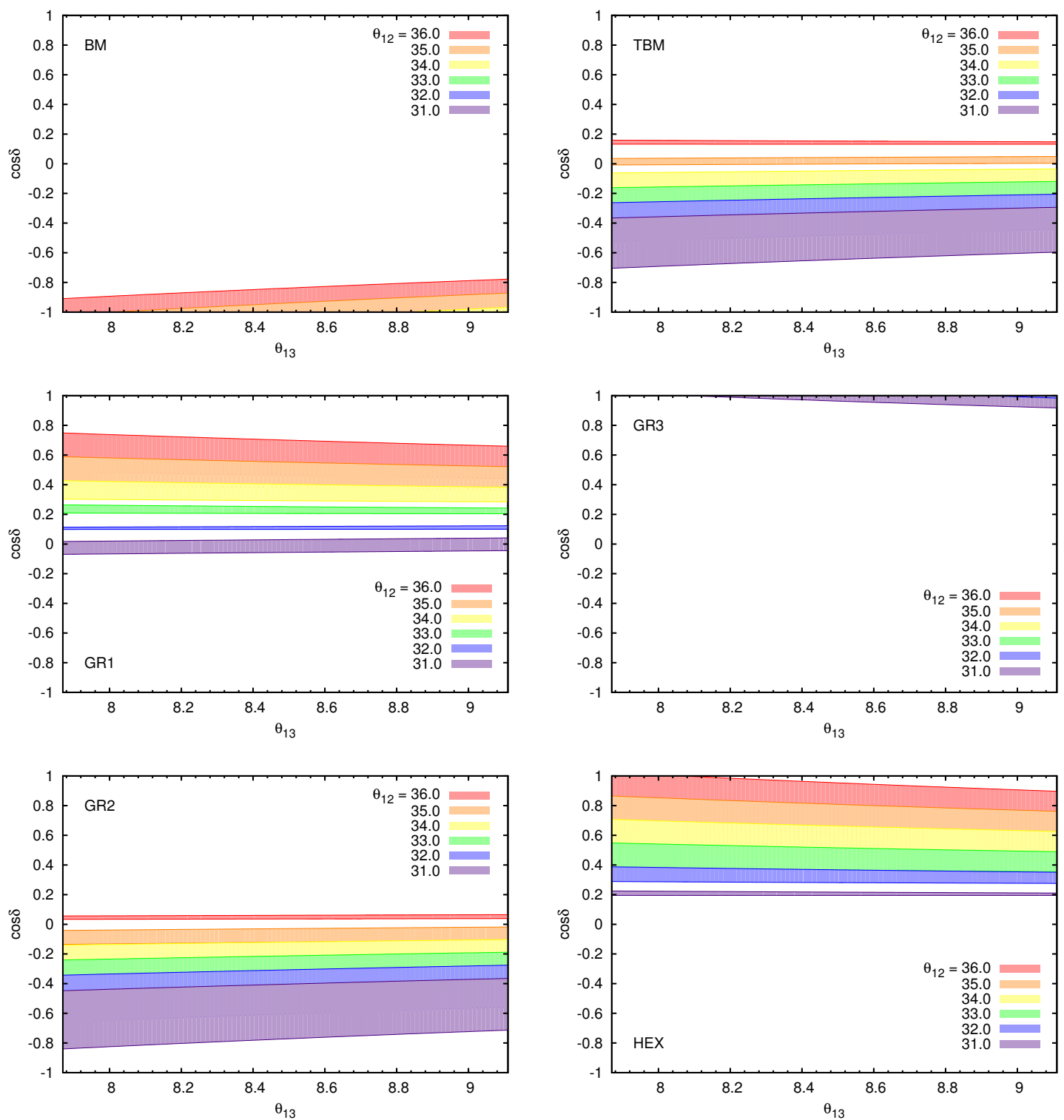

Figure 2. The predictions for $\cos \delta$ generated by the solar sum rules for BM and TBM (top row), GR1 and GR3 (middle row), GR2 and HEX (bottom row). In each plot, the true value of $\theta_{13}$ is given by the abscissa, the value of $\theta_{12}$ is denoted by the colour of the band, and the width of the band is generated by varying $\theta_{23}$ over its $3 \sigma$ allowed interval.

reasonable predictions produced by each sum rule if the parameters take values close to their current best-fits.

In summary, we find that of the four patterns well motivated by symmetry (BM, TBM, GR1 and GR3) only TBM and GR1 are consistent in a reasonable part of the parameter space. The predictions associated with BM and GR3 are only consistent in the far corners of the $3 \sigma$ intervals, where they predict maximal values of $|\cos \delta|$. For the rest of this work, we shall assume that the solar sum rules derived from BM and GR3 are excluded. 


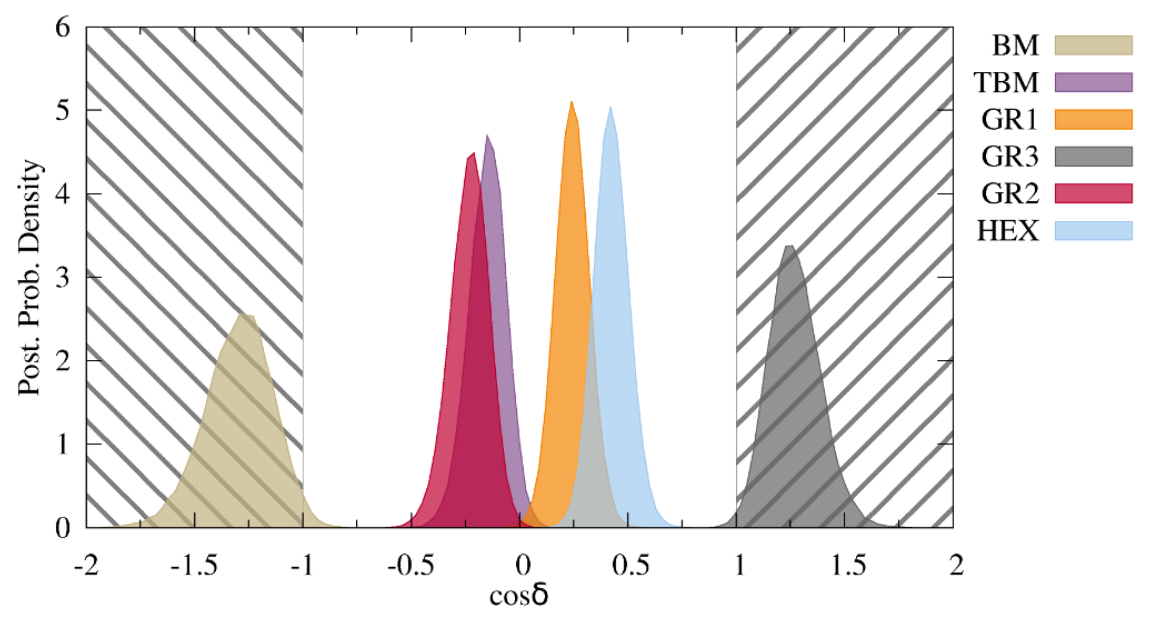

Figure 3. Posterior probability density functions for $\cos \delta$ for each of the solar sum rules considered in section 3.1. The patterned regions are unphysical, which shows that the BM and GR3 sum rules could only be consistent with the known data if there is a significant deviation from the current best-fit values.

\subsection{Simulation details}

We simulate the combination of a medium-baseline reactor (MR) experiment and a wideband superbeam (WBB). This combination of experiments is particularly interesting for the investigation of solar sum rules as MR is expected to improve the current knowledge on $\theta_{12}$, whilst the superbeam should allow $\delta$ to be constrained at a significant level for the first time. There are two proposals for a MR with comparable designs, JUNO and RENO50, and also two candidates for a next generation WBB, LBNE and LBNO. Both MRs and WBBs have similar performance targets; however, to keep our simulations concrete and relevant to experimental work, we will base our simulations on the JUNO and LBNO designs, and in this subsection we will discuss the details of our simulations of these facilties. We would like to stress that this is a purely illustrative choice, and any combination of a MR and WBB can be expected to perform similarly.

\subsubsection{JUNO}

The Jiangmen Underground Neutrino Observatory (JUNO) is a proposed reactor neutrino experiment [40], whose primary goal is to measure the neutrino mass hierarchy by observing the subtle shifts that it induces on the fast subdominant oscillations in the $\bar{\nu}_{e}$ disappearance probability [36-39]. Alongside the study of the mass hierarchy, this facility has the potential to significantly improve our measurements of $\theta_{12}, \Delta m_{21}^{2}$ and $\Delta m_{31}^{2}$ to a precision of less than $1 \%[40]$.

The JUNO experiment derives its flux from twelve nearby reactors, ten of these are at a distance of around $50 \mathrm{~km}$ from the detector with powers of either 2.9 or $4.6 \mathrm{GW}$, the remaining reactors are much further away at $215 \mathrm{~km}$ and $295 \mathrm{~km}$ both with powers 

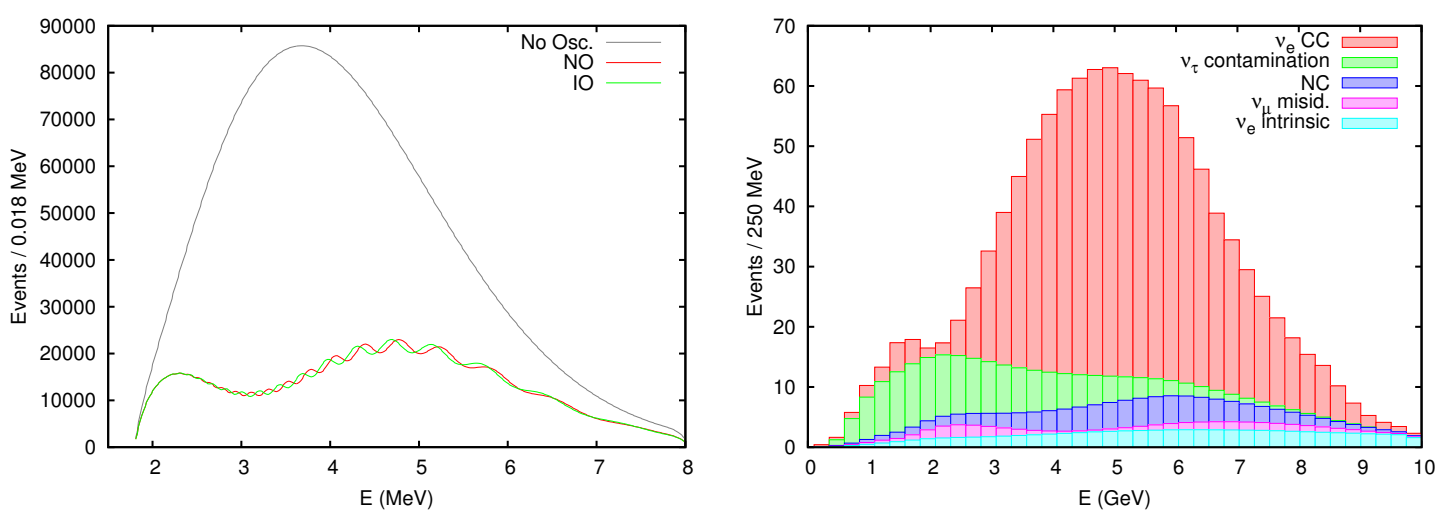

Figure 4. Example spectra from our simulations of the medium-baseline reactor experiment (left) and a wide-band superbeam (right).

of 17.4 GW [39]. JUNO's detector is assumed to be a 20 kton liquid scintillator detector. To measure the fast oscillations which generate the mass hierarchy sensitivity, JUNO must have very strong energy resolution capabilities. A linear energy dependent resolution of $\Delta E / E=0.03 / \sqrt{E / \mathrm{MeV}}$ is assumed in our simulations following the design target [40]. Non-linearities in the energy resolution are known to be a possible source of limitations for such an experiment $[37,54]$; however, as these effects are not as relevant for the precise determination of $\theta_{12}$ we assume that these effects can be controlled to a negligible level by in situ measurements, and omit them from our simulations. Our spectrum is normalised to produce $10^{5}$ total events after 6 years.

In ref. [55] it has been pointed out that the cosmogenic muon background for the next generation of large volume reactor neutrino oscillation experiments with relatively small overburdens is sufficiently large as to render the KamLAND muon cuts inapplicable whilst preserving a reasonable active period. This will be a particular problem for the delicate measurement of the neutrino mass hierarchy, but it will certainly influence the final sensitivity to $\theta_{12}$ as well. As it stands, it is unclear how the proposed experiments will circumvent this background. However, for the successful measurement of the mass hierarchy it must be addressed by some means, and in the current work, we assume that this background has been brought under control by a modification of the design or analysis. Even though this means that our work overestimates the sensitivity to $\theta_{12}$, it should not significantly alter our analysis of solar sum rules, which for the most part, only require a precision of $\theta_{12}$ at the percent level to be effective.

An illustrative event spectrum is shown in figure 4 for JUNO in both mass hierarchies (with and without oscillations). Our simulation agrees with the predicted performance in ref. [40]; in particular, our simulation provides an independent precision on $\theta_{12}$ of around $0.6 \%$.

\subsubsection{Wide-band superbeam}

A superbeam is the extrapolation of conventional neutrino beam production methods to more intense beams and larger detectors. The source neutrino beam is produced at an 
accelerator, which collides protons with a fixed target generating a spray of mesons, predominately $\pi^{ \pm}$. Magnetic focusing selects mesons of a given charge and the decay of these particles produces a beam of neutrinos. The flavour profile is mostly $\nu_{\mu}$ (for focused $\pi^{+}$) and $\bar{\nu}_{\mu}$ (for focused $\pi^{-}$); although, there is a small contamination from subdominant meson decay modes which leads to an intrinsic background of $\nu_{e}$ and $\bar{\nu}_{e}$ at the sub-percent level.

Our model of the wide-band superbeam is based upon the Long Baseline Neutrino Experiment (LBNE) [43] and Long Baseline Neutrino Oscillations (LBNO) [44] proposals for on-axis superbeams with baselines of around 1000-2000 km. The on-axis orientation ensures that the beam has a wide spectrum and allows the oscillation probability to be tested over a range of values of $L / E$, mitigating degeneracies and improving precision. Both of these experiments aim to determine the mass hierarchy and the CPV phase $\delta$ through the precise measurement of the appearance channel probabilities $P\left(\nu_{\mu} \rightarrow \nu_{e}\right)$ and $P\left(\bar{\nu}_{\mu} \rightarrow \bar{\nu}_{e}\right)$. LBNE and LBNO have a comparable physics reach, which is ultimately dependent on the precise programme of upgrades available, and we base our simulations on LBNO as described in ref. [44]. The LBNO design features as its first phase a $700 \mathrm{~kW}$ beam, a baseline distance of $2300 \mathrm{~km}$ between CERN and the Pyhäsalmi mine in Finland, and a 20 kton detector based on liquid argon time-projection chamber technology [44, 56]. Our simulation of this facility uses the fluxes provided by ref. [57], and propagates the neutrinos through a constant density background of $3.2 \mathrm{~g} / \mathrm{cm}^{3}$. We consider both the appearance $\nu_{\mu} \rightarrow \nu_{e}\left(\bar{\nu}_{\mu} \rightarrow \bar{\nu}_{e}\right)$ and muon disappearance channels $\nu_{\mu} \rightarrow \nu_{\mu}\left(\bar{\nu}_{\mu} \rightarrow \bar{\nu}_{\mu}\right)$. The background to the appearance channel is given by the intrinsic $\nu_{e}$ component of the beam, misidentified $\nu_{\mu}$ events at a rate of $1 \%, 2 \%$ of neutral current events and events arising from $\tau$-contamination: the production of $\tau^{ \pm}$leptons in the detector which quickly decay to $e^{ \pm}$. These $\tau$ events have been implemented via a custom migration matrix which maps the spectrum of incoming $\nu_{\mu}\left(\bar{\nu}_{\mu}\right)$ onto the resultant $e^{-}\left(e^{+}\right)$post-decay distribution. We have normalised our number of events to match the tables simulated in ref. [44], which assumes a total of $10^{21}$ protons on target corresponding roughly to 10 years of run time, but we consider masses of 35 kton and 70 kton to account for a reasonable range of possible detectors, according to the LBNE and LBNO phased designs and upgrade programmes [43, 44]. An illustrative spectrum decomposed into its background components can be seen in the right panel of figure 4 . We see a close agreement of form for most of our backgrounds when our spectrum is compared with the spectra in ref. [44]. The only notable deviation is in the shape of our neutral current background, but this small difference is not expected to effect the general conclusions of the current work.

\subsection{Simulation results}

Our simulation combines the expected data from a long-baseline wide-band superbeam (WBB) experiment, modelled after LBNO although it also provides a good estimate of the performance of LBNE, and a medium-baseline reactor (MR) experiment with a baseline around $60 \mathrm{~km}$, modelled on JUNO. As discussed previously, these facilities are expected to provide complementary constraints on the parameters relevant for the solar sum rule. This synergy can be seen in figure 5 , where we show the independent constraints on $\delta$ and 


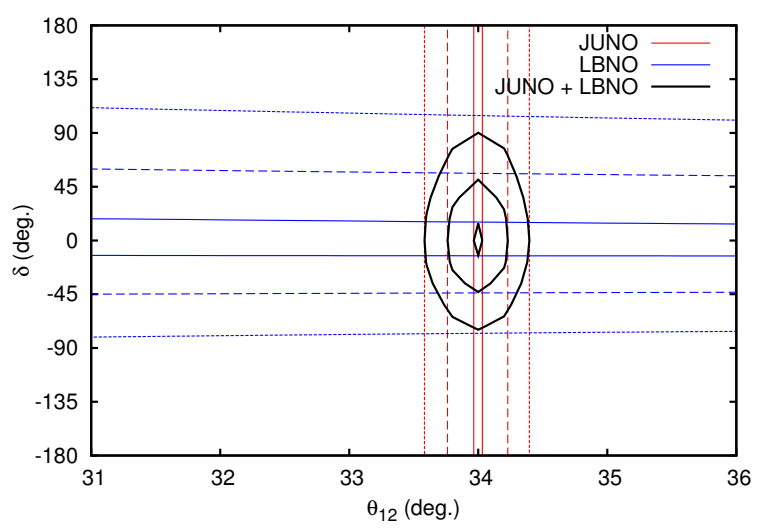

Figure 5. The independent and combined constraints on the parameters $\delta$ and $\theta_{12}$ for an illustrative point in parameter space for the JUNO and LBNO experiments. In each experimental arrangement, the three lines correspond to 1,3 and $5 \sigma$ significance.

$\theta_{12}$ provided by the LBNO and JUNO experiments and their combination. As expected, the precision is dominated by JUNO for $\theta_{12}$ and by LBNO for $\delta$.

To determine the allowed regions for a given sum rule we generate the expected event rates for each set of true parameters of interest. We then compute the set of hypothetical parameters which maximise the likelihood function under two different hypotheses: first, assuming no constraints on the parameter space, which finds the best-fitting point, and secondly, whilst imposing the constraint of the sum rule on our hypothesised parameters. When the constrained best-fit has a likelihood significantly below the unconstrained bestfit, we conclude that the sum rule can be excluded. During the maximisation process, we include priors on the oscillation parameters to account for the external constraints of the global data. If not mentioned explicitly, we assume the following values for the true parameters

$$
\theta_{12}=33.48^{\circ}, \quad \theta_{13}=8.50^{\circ} \text { and } \theta_{23}=42.3^{\circ},
$$

and the mass splittings

$$
\Delta m_{21}^{2}=7.50 \times 10^{-5} \mathrm{eV}^{2} \text { and } \Delta m_{31}^{2}=2.46 \times 10^{-3} \mathrm{eV}^{2} .
$$

For the prior constraints, we assume a $2 \%(2.4 \%)$ uncertainty on $\Delta m_{31}^{2}\left(\Delta m_{21}^{2}\right)$ and uncertainties of $2.3 \%, 2.4 \%$ and $6 \%$ on $\theta_{12}, \theta_{13}$ and $\theta_{23}$, respectively. These are chosen to be in agreement with recent best-fits [3]. We assume normal hierarchy in all of our simulations, although it has been checked that the results are not particularly sensitive to this choice. Our simulations are implemented using the GLoBES package $[58,59]$.

In figure 6, we show the results of our simulations of MR and WBB for the measurement of the solar sum rules defined by eq. (1.6). The four models shown are the tribimaximal $(\mathrm{TBM})$ model with $\theta_{12}^{\nu}=\arcsin \frac{1}{\sqrt{3}}$, the golden ratio model with $\theta_{12}^{\nu}=\arctan \frac{1}{\varphi}$, and the two dihedral models, GR2 and HEX, with $\theta_{12}^{\nu}=\frac{\pi}{5}$ and $\theta_{12}^{\nu}=\frac{\pi}{6}$, respectively. In the left panel on the top row, we see the regions of true parameter space for which the TBM model cannot be excluded at 2 and $3 \sigma$ significance. As we saw in figure 2, all four of the models under consideration here make consistent predictions for all values of $\theta_{12}$. The plots predict 
a similar fraction of true parameter space in which these models can be excluded: at $2 \sigma$ the models can be excluded in around $65 \%$ of the parameter space, while at $3 \sigma$ this drops to around $26 \%$. These plots show only a mild dependence on the true value of $\theta_{12}$. Comparing the panels on the top row, which show the two models well motivated by symmetry, we see that the smaller value of $\theta_{12}^{\nu}$ in the GR1 model compared to the TBM prediction generates a smaller prediction for $|\delta|$. Although, there is significant overlap between the allowed regions of the two models, which means it is unlikely that they could be distinguished with the 35 kton detector. The bottom row shows the two dihedral predictions (GR2 on the left, and HEX on the right). For HEX, the smaller value of $|\delta|$ leads to the $3 \sigma$ regions merging at $\delta \approx 0$. In practice, this means that for larger values of $\theta_{12}$, it is easier to exclude the model: for $\theta_{12}=35.3^{\circ}$, we see the fraction of parameter space for which the model can be excluded increase to around $38 \%$ at $3 \sigma$.

Although distinguishing between these models will be challenging, each can be excluded for a reasonable region of the parameter space through the combination of data from a MR and WBB experiment. In general, we can also point out that an observation of an extreme value of $\cos \delta$ would allow all of these models to be excluded: a true value of $|\cos \delta|=1$ or $\cos \delta=0$ would disfavour all models at $2 \sigma$ and for most of the parameter space exclude them at $3 \sigma$.

Upgrading the WBB experiment would allow the discovery potential to be significantly extended. In figure 7, we show the effect of increasing the detector mass to $70 \mathrm{kton}$. This doubling of statistics allows the precision on $\delta$ to increase, which leads to larger exclusion areas. We show the results for TBM and GR1, where the exclusion regions are now around $71 \%$ of the true parameter space at $2 \sigma$ and $48 \%$ at $3 \sigma$. This corresponds to an $80 \%$ increase in the $3 \sigma$ exclusion region. Clearly, to fully understand the potential for this measurement, the foreseen programme of upgrades will play an important role.

\section{Beyond $\theta_{13}^{\nu}=0$}

Neutrino mixing patterns in which $\theta_{13}^{\nu} \neq 0$ have also been predicted in the literature, and can likewise give rise to solar sum rules. However, eq. (1.6) does not apply in such scenarios, and we must work on a case by case basis. Examples of patterns of this type can be found in the fully-specified patterns in ref. [52]. Of these patterns, we identify 10 unique mixing matrices from the sporadic patterns which meet our criteria on the mixing angles and have $\theta_{13}^{\nu} \neq 0$, along with an infinite subset of the family $\mathcal{C}_{2}$.

As an example, we shall derive another sum rule from one member of $\mathcal{C}_{2}$ characterised by a purely imaginary parameter $\sigma=i$, cf. eq. (150) of ref. [52]. This pattern had been previously studied in a grand-unified model of flavour based on the group $\Delta(96) \times \mathrm{SU}(5)$ [60], where it was known as the bi-trimaximal mixing (BTM) pattern,

$$
U_{\mathrm{BTM}}^{\nu}=\left(\begin{array}{ccc}
a_{+} & \frac{1}{\sqrt{3}} & a_{-} \\
-\frac{1}{\sqrt{3}} & \frac{1}{\sqrt{3}} & \frac{1}{\sqrt{3}} \\
a_{-} & -\frac{1}{\sqrt{3}} & a_{+}
\end{array}\right),
$$



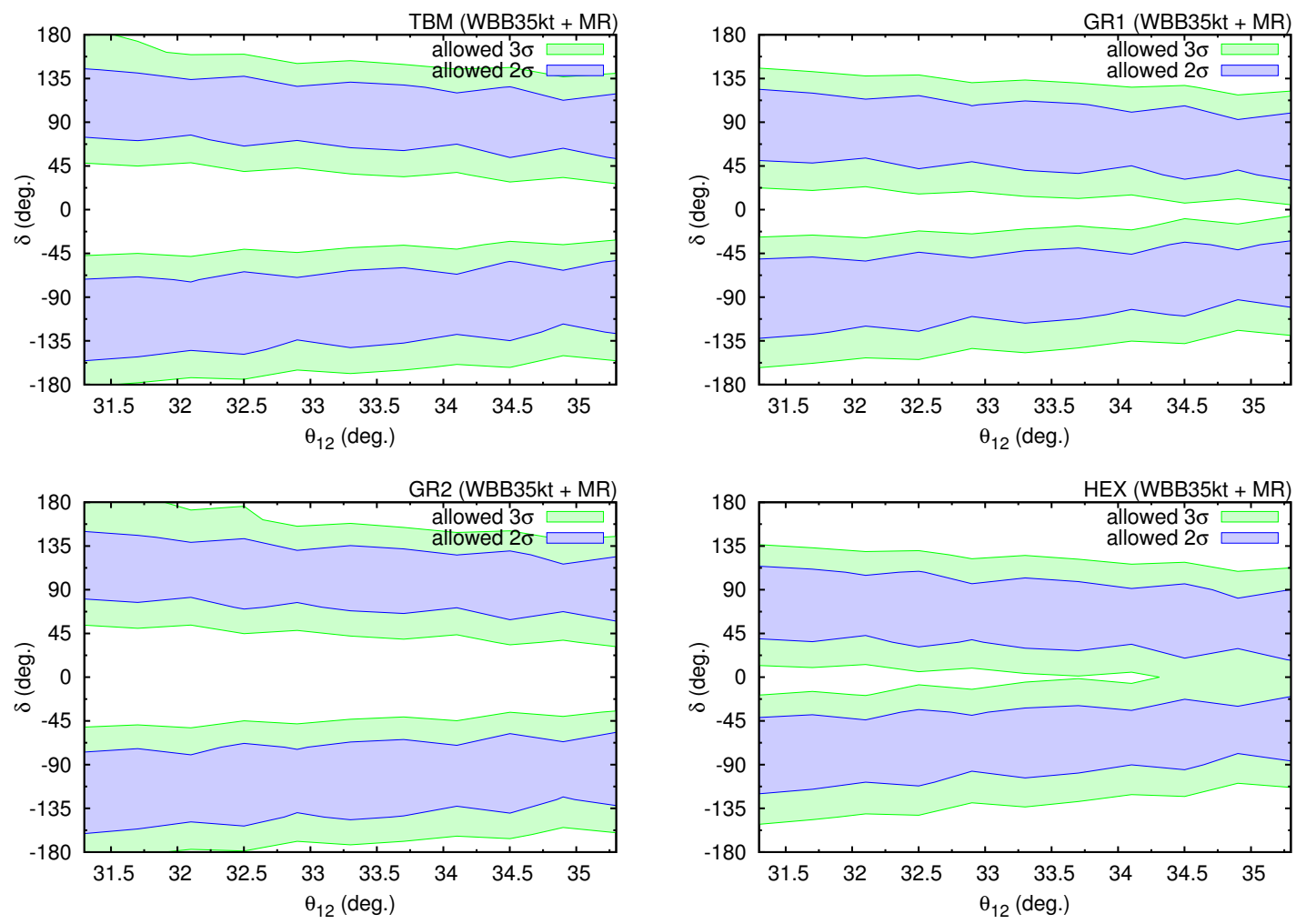

Figure 6. The allowed regions of true parameter space in the $\theta_{12}-\delta$ plane for TBM (left, top row), GR1 (right, top row), GR2 (left, bottom row) and HEX (right, bottom row) after 6 years of data taken by a medium-baseline reactor experiment (MR) and 10 years by a wide-band superbeam with 35 kton detector (WBB35kt).
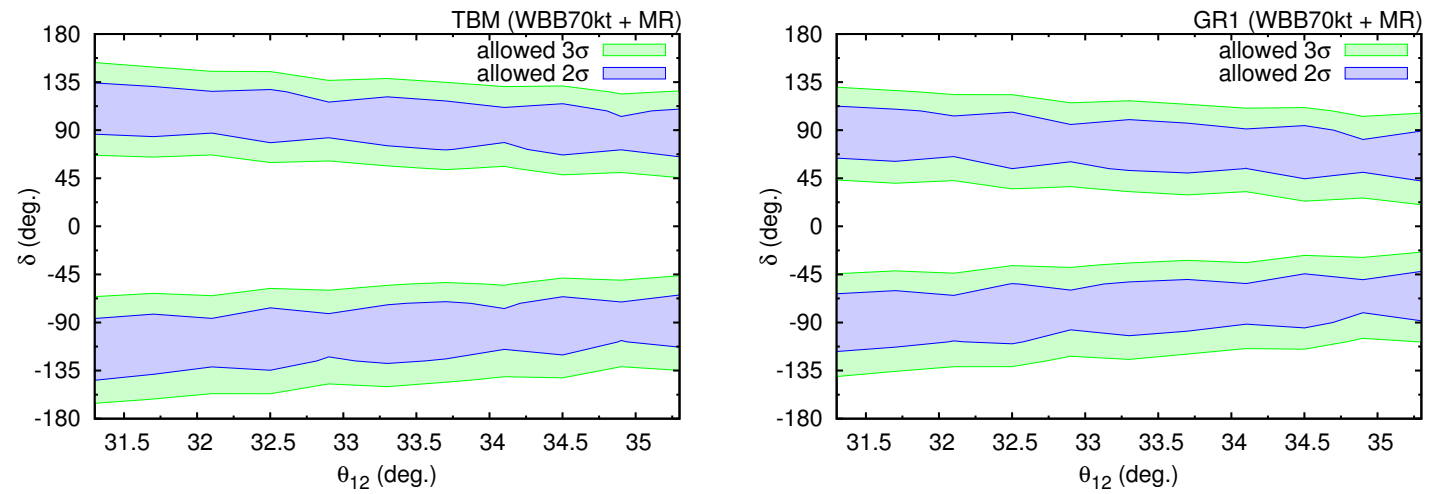

Figure 7. The allowed regions of true parameter space in the $\theta_{12}-\delta$ plane for TBM (left) and GR1 (right) after 6 years of data taken by a medium-baseline reactor experiment (MR) and 10 years by a wide-band superbeam with an upgraded 70 kton detector (WBB70kt). 
with $a_{ \pm}=\left(1 \pm \frac{1}{\sqrt{3}}\right) / 2$. Multiplication of the charged lepton mixing $U_{12}^{e}{ }^{\dagger}$ from the left yields the PMNS matrix (up to Majorana and unphysical phases)

$$
U=\left(\begin{array}{ccc}
a_{+} c_{12}^{e}+\frac{1}{\sqrt{3}} s_{12}^{e} e^{-i \delta_{12}^{e}} & \frac{1}{\sqrt{3}} c_{12}^{e}-\frac{1}{\sqrt{3}} s_{12}^{e} e^{-i \delta_{12}^{e}} & a_{-} c_{12}^{e}-\frac{1}{\sqrt{3}} s_{12}^{e} e^{-i \delta_{12}^{e}} \\
a_{+} s_{12}^{e} e^{i \delta_{12}^{e}-\frac{1}{\sqrt{3}} c_{12}^{e}} & \frac{1}{\sqrt{3}} s_{12}^{e} e^{i \delta_{12}^{e}+\frac{1}{\sqrt{3}} c_{12}^{e}} & a_{-} s_{12}^{e} e^{i \delta_{12}^{e}}+\frac{1}{\sqrt{3}} c_{12}^{e} \\
a_{-} & -\frac{1}{\sqrt{3}} & a_{+}
\end{array}\right)
$$

The two free continuous parameters $\theta_{12}^{e}$ and $\delta_{12}^{e}$ will control the four physical parameters $\theta_{12}, \theta_{13}, \theta_{23}$ and $\delta$. Therefore we expect two sum rules. They can be derived easily by comparing the (square of the) absolute value of the $(i, j)$ entry with the corresponding entry in the PDG parameterisation of $U$. From $U_{\tau 3}$ we find the first exact sum rule involving the atmospheric angle

$$
c_{13} c_{23}=a_{+} .
$$

Similarly we get from $U_{\tau 2}$

$$
c_{23}^{2} s_{12}^{2} s_{13}^{2}+s_{23}^{2} c_{12}^{2}+2 s_{23} c_{23} s_{12} c_{12} s_{13} \cos \delta=\frac{1}{3} .
$$

Solving eq. (4.3) for $\theta_{23}$ and inserting the result into eq. (4.4) gives rise to the sum rule involving $\theta_{12}, \theta_{13}$ and $\delta$,

$$
\cos \delta=\frac{c_{13}^{2}-3 a_{+}^{2} s_{12}^{2} s_{13}^{2}-3 c_{12}^{2}\left(c_{13}^{2}-a_{+}^{2}\right)}{6 a_{+} s_{12} c_{12} s_{13} \sqrt{c_{13}^{2}-a_{+}^{2}}} .
$$

Satisfying both of these constraints simultaneously is very difficult with the known global data on the mixing angles: $\cos \delta$ is only well defined for large values of $\theta_{13}$ and small values of $\theta_{12}$, but the constraint of eq. (4.3) requires that $\theta_{13}$ and $\theta_{23}$ are at the low-valued extremes of their allowed parameter space. This tension may be alleviated by introducing further corrections to these predictions, for example the renormalisation effects.

\section{Renormalisation group corrections}

In this analysis we have ignored the effects of renormalisation group (RG) corrections to mixing angles. Although this is generally a good approximation, it is useful to be aware of the typical magnitudes of such corrections and when they might be important. In this section, we briefly review such issues. For previous discussion of RG corrections in this context see e.g. ref. [61] for a discussion in case of Cabibbo-like charged-lepton correction to BM mixing and charged-lepton corrections to TBM in ref. [62].

In the effective theory the RG correction to $\theta_{12}$, which is generically the largest, is described by the following renormalisation group equation [63]

$$
\dot{\theta}_{12} \equiv \frac{d \theta_{12}}{d \ln \left(\mu / \mu_{0}\right)}=-\frac{C y_{\tau}^{2}}{32 \pi^{2}} \sin 2 \theta_{12} s_{23}^{2} \frac{\left|m_{1} e^{i \varphi_{1}}+m_{2} e^{i \varphi_{2}}\right|^{2}}{m_{2}^{2}-m_{1}^{2}}+\mathcal{O}\left(\theta_{13}\right),
$$

with $C=1$ in the Minimal Supersymmetric Standard Model (MSSM) and $C=-3 / 2$ in the Standard Model (SM). In the MSSM large values of $\tan \beta=v_{u} / v_{d}$ lead to an enhancement 
of the RG running via the Yukawa coupling $y_{\tau}^{2}=m_{\tau}^{2}\left(1+\tan ^{2} \beta\right) / v^{2}$, where $v=246 \mathrm{GeV}$. There is no such enhancement in the SM. In order to estimate the size of the RG corrections, we use the exact analytic RG equation for $\theta_{12}$ fixing all parameters at their respective best fit values. We do not include the running of the other parameters. The running of the solar mixing angle from the electroweak scale to the GUT scale $\Lambda_{\mathrm{GUT}}=2 \cdot 10^{16} \mathrm{GeV}$ is given to leading order by $\Delta \theta_{12}=\theta_{12}\left(\Lambda_{\mathrm{GUT}}\right)-\theta_{12}\left(m_{Z}\right) \simeq \dot{\theta}_{12}\left(m_{Z}\right) \ln \left(2 \cdot 10^{14}\right)$. It depends on the absolute neutrino mass scale.

In the SM the corrections are generally small. Using the exact ${ }^{6}$ one-loop formula for $\theta_{12}$ given in ref. [63], we obtain a conservative estimate by taking a quasi-degenerate mass spectrum with vanishing phases: $\Delta \theta_{12} \approx 0.17^{\circ}\left(0.04^{\circ}\right)$ for $m_{1}=0.1 \mathrm{eV}\left(m_{1}=\sqrt{\Delta m_{32}^{2}}\right)$. Experimentally, the expected sensitivity for determining $\theta_{12}$ is about $0.6 \%$ [40], i.e. $\Delta \theta_{12} \approx$ $0.2^{\circ}$. In the context of the SM it is therefore justified to neglect RG effects on the solar sum rules discussed in this paper.

On the other hand, RG corrections are typically bigger in the MSSM. We have considered 4 different cases: normal and inverted mass ordering and a Majorana phase difference $\Delta \varphi=\varphi_{2}-\varphi_{1}$ of 0 and $\pi$. With $\Delta \varphi=0$, RG effects become more and more relevant for increasing absolute neutrino mass scales and increasing values of $\tan \beta$. For an inverted mass ordering, the lower bound on $m_{1}$ entails relevant $\mathrm{RG}$ corrections for $\tan \beta \gtrsim 2$. In the case of a normal mass ordering, $\mathrm{RG}$ corrections have to be taken into account if $\tan \beta \gtrsim 45$, where this bound is decreasing with increasing $m_{1}$, e.g. with $m_{1} \approx \sqrt{\Delta m_{21}^{2}}$ one has $\tan \beta \gtrsim 10$. A non-vanishing Majorana phase difference generically suppresses RG running. For $\Delta \varphi=\pi$, the leading term in eq. (5.1) is proportional to $\Delta m_{21}^{2} /\left(m_{1}+m_{2}\right)^{2}$, such that it decreases with increasing absolute neutrino mass scale. RG corrections to the solar sum rules can be neglected for $\tan \beta \lesssim 35$ provided the neutrino mass spectrum is not quasi-degenerate.

\section{Conclusion}

We have presented a succinct derivation of solar lepton mixing sum rules, arising from simple patterns of neutrino mixing with $\theta_{13}^{\nu}=0$, enforced by discrete family symmetry and corrected by a rather generic charged lepton mixing matrix, assuming only that $\theta_{13}^{e}=0$. From our derivation we have expressed the result as the ratio of the absolute magnitude of two PMNS matrix elements, given in terms of $\theta_{12}^{\nu}$, namely $\left|U_{\tau 1}\right| /\left|U_{\tau 2}\right|=t_{12}^{\nu}$. When expanded in terms of the three PMNS mixing angles and the CPV oscillation phase $\delta$, the resulting solar sum rule may be cast in terms of a prediction for $\cos \delta$ which depends only on $\theta_{12}^{\nu}$.

We have considered in detail the resulting solar mixing sum rules, arising from four particularly well-motivated cases of neutrino mixing, which can be derived from discrete family symmetries, namely: BM mixing where $s_{12}^{\nu}=1 / \sqrt{2}$, TBM mixing where $s_{12}^{\nu}=1 / \sqrt{3}$, and two patterns based on versions of golden ratio mixing including GR1 with $t_{12}^{\nu}=1 / \varphi$ and GR3 with $c_{12}^{\nu}=\varphi / \sqrt{3}$, where $\varphi=(1+\sqrt{5}) / 2$ is the golden ratio. We have also fully

\footnotetext{
${ }^{6}$ It turns out that the term proportional to $\theta_{13}$ becomes relevant in eq. (5.1) and the exact expression of $\dot{\theta}_{12}$ has to be used.
} 
discussed two leading-order patterns which have been invoked in the literature, but which cannot be enforced by any simple discrete family symmetry, called GR2 with $\theta_{12}^{\nu}=\pi / 5$ and HEX mixing with $\theta_{12}^{\nu}=\pi / 6$.

It turns out that two of the above six cases, namely BM and GR3, are almost excluded by current data, so in the phenomenological study, we have focused on the remaining four viable cases, namely TBM, GR1, GR2 and HEX, of which only the first two (TBM and GR1) are well founded by symmetry arguments. The predictions for $\cos \delta$ for all these cases are summarised in figure 3 . For the four viable cases, we performed a simulation of a next-generation superbeam experiment, based on LBNO, and a future reactor experiment, based on JUNO, to see how well the sum rules can be tested. For example, in figure 6 we show the allowed regions of true parameter space in the $\theta_{12}-\delta$ plane, following a 6 -year medium-baseline reactor experiment and a decade of running with a WBB and a 35 kton detector.

We have seen that the ability to constrain solar sum rules relies crucially on the complementary sensitivities of both reactor and superbeam experiments, and that, acting together, these facilities will be capable of significantly restricting the allowed parameter space of the models associated with solar mixing sum rules. It is possible that such experiments could exclude all of the models considered in this paper, which would be the case if, for example $\delta \approx 0$. If this occurs, the theoretical approach of explaining lepton mixing as a result of charged-lepton corrections to simple symmetry-driven patterns of neutrino mixing would become strongly disfavoured.

\section{Acknowledgments}

We acknowledge support from the European Union FP7 ITN-INVISIBLES (Marie Curie Actions, PITN- GA-2011-289442). The work of CL is supported by the Deutsche Forschungsgemeinschaft within the Research Unit FOR 1873 (Quark Flavour Physics and Effective Field Theories). MS acknowledges support by the Australian Research Council. This work has been additionally supported by the European Research Council under ERC Grant "NuMass" (FP7-IDEAS-ERC ERC-CG 617143).

\section{A Simple approximation to the mixing sum rule}

In this appendix we show that the exact sum rule in eq. (1.6) reduces to the well-known leading-order sum rule to an accuracy of a few percent. If we drop terms proportional to $s_{13}^{2}$ in eq. (1.6), we obtain the approximate sum rule,

$$
\cos \delta \approx \frac{t_{23}\left(s_{12}^{2}-s_{12}^{\nu 2}\right)}{\sin 2 \theta_{12} s_{13}} .
$$

This sum rule can be written to leading order in $\theta_{13}$ as,

$$
\frac{s_{12}^{2}-s_{12}^{\nu 2}}{2 s_{12} c_{12}} \approx \frac{\theta_{13}}{t_{23}} \cos \delta .
$$


If we write $\theta_{12}=\theta_{12}^{\nu}+\varepsilon_{12}$, then to leading order in $\varepsilon_{12}$,

$$
\frac{s_{12}^{2}-s_{12}^{\nu 2}}{2 s_{12} c_{12}} \approx \varepsilon_{12} .
$$

Hence eq. (A.1) becomes, to leading order in $\varepsilon_{12}$ and $\theta_{13}$,

$$
\theta_{12}-\theta_{12}^{\nu} \approx \frac{\theta_{13}}{t_{23}} \cos \delta
$$

If we write $\theta_{23}=\pi / 4+\varepsilon_{23}$, then to leading order in $\varepsilon_{23}, \varepsilon_{12}$ and $\theta_{13}$ we find,

$$
\theta_{12}-\theta_{12}^{\nu} \approx \theta_{13} \cos \delta
$$

which is the usual well-known leading-order solar sum rule $[8,20,21]$. The corrections to this linearised sum rule are of order $\theta_{13}^{2}, \varepsilon_{12}^{2}$ and $\theta_{13} \varepsilon_{23}$. For example, the second order correction from the reactor angle is $\theta_{13}^{2} \sim(0.15)^{2} \sim 0.02 \sim 2 \%$. The formulae preceding eq. (A.3) may be used for a more accurate approximate description of the sum rule. For example, eq. (A.2) could be used to better account for the atmospheric mixing angle deviating from maximal.

\section{B Neutrino mixing patterns from $\mathbb{Z}_{2} \times \mathbb{Z}_{2}$}

The possibility that the full $\mathbb{Z}_{2} \times \mathbb{Z}_{2}$ symmetry of the neutrino mass term is a subgroup of the flavour symmetry was considered from a bottom-up perspective in ref. [13]. It is a simple extension of the authors' previous constraints (see ref. [12] for details): each neutrino generator $S_{i}$ fixes one column of the mixing matrix using the formulae,

$$
\begin{aligned}
\cos ^{2}\left(\frac{\pi d}{p}\right) & =\sin ^{2}\left(\frac{\pi k}{m}\right)\left|U_{\alpha i}^{\nu}\right|^{2}, \\
0 & =\sin \left(\frac{2 \pi k}{m}\right)\left(\left|U_{\beta i}^{\nu}\right|^{2}-\left|U_{\gamma i}^{\nu}\right|^{2}\right),
\end{aligned}
$$

where $k, m, d, p \in \mathbb{N}$ such that $0<k<m$ and $0<d<p$ with the requirement that $k$ and $m(d$ and $p)$ are coprime, and $\{\alpha, \beta, \gamma\}=\{e, \mu, \tau\}$. Neglecting for the time being the case $m=2$ or $p=2$, the constraint of unitarity implies that fixing two columns of the mixing matrix using these formulae fully specifies the pattern. Each column is given by a permutation of $\left\{\sqrt{\eta}, \sqrt{\frac{1-\eta}{2}}, \sqrt{\frac{1-\eta}{2}}\right\}$, where the $\eta$ parameter for the first and second column are given, respectively, by

$$
\eta_{1}=\frac{\cos ^{2}\left(\frac{\pi d_{1}}{p_{1}}\right)}{\sin ^{2}\left(\frac{\pi k_{1}}{m_{1}}\right)} \quad \text { and } \quad \eta_{2}=\frac{\cos ^{2}\left(\frac{\pi d_{2}}{p_{2}}\right)}{\sin ^{2}\left(\frac{\pi k_{2}}{m_{2}}\right)} .
$$

We can generate the consistent mixing patterns by choosing those $k_{i}, m_{i}, p_{i}$ and $d_{i}$ parameters which generate finite groups. Considering only patterns related to von Dyck groups with irreducible triplets, i.e. $\mathrm{A}_{4}, \mathrm{~S}_{4}$ and $\mathrm{A}_{5}$, the result is the set of numbers listed in eq. (2.11). As we are assuming a single $\mathbb{Z}_{m}$ residual symmetry in the charged-lepton sector, we must also take a common value of $m$, i.e. $m_{1}=m_{2}$. 
Up to row and column permutations, there are two ways of relatively aligning the elements of the two fixed columns. In one case, we choose the unique element fixed by the two real constraints to be in the same row,

$$
\left|U_{\alpha i}^{\nu}\right|^{2}=\left(\begin{array}{ccc}
\eta_{1} & \eta_{2} & 1-\eta_{1}-\eta_{2} \\
\frac{1-\eta_{1}}{2} & \frac{1-\eta_{2}}{2} & \frac{\eta_{1}+\eta_{2}}{2} \\
\frac{1-\eta_{1}}{2} & \frac{1-\eta_{2}}{2} & \frac{\eta_{1}+\eta_{2}}{2}
\end{array}\right) .
$$

In the other case, we choose these elements to be misaligned,

$$
\left|U_{\alpha i}^{\nu}\right|^{2}=\left(\begin{array}{ccc}
\eta_{1} & \frac{1-\eta_{2}}{2} & \frac{1}{2}-\eta_{1}+\frac{\eta_{2}}{2} \\
\frac{1-\eta_{1}}{2} & \eta_{2} & \frac{1}{2}-\eta_{2}+\frac{\eta_{1}}{2} \\
\frac{1-\eta_{1}}{2} & \frac{1-\eta_{2}}{2} & \frac{\eta_{1}+\eta_{2}}{2}
\end{array}\right) .
$$

However, patterns of the form given in eq. (B.3) are not possible if the residual symmetry in the charged-lepton sector has only a single generator: it is the choice of $T_{\alpha}$ which specifies the row $\alpha$ of the $\eta$ parameter. Under our assumption of a single cyclic charged-lepton symmetry, $\mathbb{Z}_{m}$, we only need to consider patterns of the form of eq. (B.2) and its row and column permutations.

Up to now, we have refrained from discussing the case related to dihedral groups where $\{m, p\}=\{2, N\}$. In fact, such a scenario does not give rise to any new eligible neutrino patterns. If $m$ or $p$ take the value 2, the symmetry constraints from the generators of this subgroup in eq. (B.1) do not necessarily fix a column of matrix elements, leaving parts of the leading-order mixing matrix unspecified. Therefore, we will only consider the choice which does indeed fix a column completely; it is given by $(m, p)=(N, 2)$ with $N>2$ and yields

$$
U_{\alpha i}^{\nu}=0 \quad \text { and } \quad\left|U_{\beta i}^{\nu}\right|=\left|U_{\gamma i}^{\nu}\right| .
$$

Then, the only way to fully specify the mixing matrix is to either apply this constraint twice, or to apply this constraint in conjunction with one related to $\mathrm{A}_{4}, \mathrm{~S}_{4}$ or $\mathrm{A}_{5}$. By scanning through the row and column permutations of eq. (B.2), we find that the only viable choices are (unsurprisingly) when the dihedral constraint is applied once and is used to fix $U_{e 3}^{\nu}=0$; however, all patterns of this type reproduce patterns already discussed previously.

Open Access. This article is distributed under the terms of the Creative Commons Attribution License (CC-BY 4.0), which permits any use, distribution and reproduction in any medium, provided the original author(s) and source are credited.

\section{References}

[1] DAYA-BAY collaboration, F.P. An et al., Observation of electron-antineutrino disappearance at Daya Bay, Phys. Rev. Lett. 108 (2012) 171803 [arXiv:1203.1669] [INSPIRE].

[2] RENO collaboration, J.K. Ahn et al., Observation of Reactor Electron Antineutrino Disappearance in the RENO Experiment, Phys. Rev. Lett. 108 (2012) 191802 [arXiv: 1204.0626] [INSPIRE]. 
[3] M.C. Gonzalez-Garcia, M. Maltoni and T. Schwetz, Updated fit to three neutrino mixing: status of leptonic CP-violation, JHEP 11 (2014) 052 [arXiv:1409.5439] [INSPIRE].

[4] D.V. Forero, M. Tortola and J.W.F. Valle, Neutrino oscillations refitted, Phys. Rev. D 90 (2014) 093006 [arXiv: 1405.7540] [INSPIRE].

[5] F. Capozzi et al., Status of three-neutrino oscillation parameters, circa 2013, Phys. Rev. D 89 (2014) 093018 [arXiv: 1312.2878] [InSPIRE].

[6] P.F. Harrison, D.H. Perkins and W.G. Scott, Tri-bimaximal mixing and the neutrino oscillation data, Phys. Lett. B 530 (2002) 167 [hep-ph/0202074] [INSPIRE].

[7] S.F. King and C. Luhn, On the origin of neutrino flavour symmetry, JHEP 10 (2009) 093 [arXiv:0908.1897] [INSPIRE].

[8] S.F. King, Predicting neutrino parameters from $\mathrm{SO}(3)$ family symmetry and quark-lepton unification, JHEP 08 (2005) 105 [hep-ph/0506297] [INSPIRE].

[9] S.F. King and C. Luhn, Neutrino Mass and Mixing with Discrete Symmetry, Rept. Prog. Phys. 76 (2013) 056201 [arXiv: 1301.1340] [INSPIRE].

[10] S.F. King, A. Merle, S. Morisi, Y. Shimizu and M. Tanimoto, Neutrino Mass and Mixing: from Theory to Experiment, New J. Phys. 16 (2014) 045018 [arXiv:1402.4271] [InSPIRE].

[11] S.F. King, Parametrizing the lepton mixing matrix in terms of deviations from tri-bimaximal mixing, Phys. Lett. B 659 (2008) 244 [arXiv:0710.0530] [INSPIRE].

[12] D. Hernandez and A.Y. Smirnov, Lepton mixing and discrete symmetries, Phys. Rev. D 86 (2012) 053014 [arXiv: 1204.0445] [INSPIRE].

[13] D. Hernandez and A.Y. Smirnov, Discrete symmetries and model-independent patterns of lepton mixing, Phys. Rev. D 87 (2013) 053005 [arXiv: 1212.2149] [INSPIRE].

[14] P. Ballett, S.F. King, C. Luhn, S. Pascoli and M.A. Schmidt, Testing atmospheric mixing sum rules at precision neutrino facilities, Phys. Rev. D 89 (2014) 016016 [arXiv:1308.4314] [INSPIRE].

[15] Y. Shimizu, M. Tanimoto and A. Watanabe, Breaking Tri-bimaximal Mixing and Large $\theta_{13}$, Prog. Theor. Phys. 126 (2011) 81 [arXiv:1105.2929] [INSPIRE].

[16] S.F. King and C. Luhn, Trimaximal neutrino mixing from vacuum alignment in A4 and $S_{4}$ models, JHEP 09 (2011) 042 [arXiv: 1107.5332] [INSPIRE].

[17] S. Antusch, S.F. King, C. Luhn and M. Spinrath, Trimaximal mixing with predicted $\theta_{13}$ from a new type of constrained sequential dominance, Nucl. Phys. B 856 (2012) 328 [arXiv: 1108.4278] [INSPIRE].

[18] S.F. King, Minimal predictive see-saw model with normal neutrino mass hierarchy, JHEP 07 (2013) 137 [arXiv: 1304.6264] [INSPIRE].

[19] S.F. King, Minimal see-saw model predicting best fit lepton mixing angles, Phys. Lett. B 724 (2013) 92 [arXiv: 1305.4846] [INSPIRE].

[20] I. Masina, A Maximal atmospheric mixing from a maximal CP-violating phase, Phys. Lett. B 633 (2006) 134 [hep-ph/0508031] [INSPIRE].

[21] S. Antusch and S.F. King, Charged lepton corrections to neutrino mixing angles and CP phases revisited, Phys. Lett. B 631 (2005) 42 [hep-ph/0508044] [INSPIRE]. 
[22] S. Antusch, P. Huber, S.F. King and T. Schwetz, Neutrino mixing sum rules and oscillation experiments, JHEP 04 (2007) 060 [hep-ph/0702286] [INSPIRE].

[23] D. Marzocca, S.T. Petcov, A. Romanino and M.C. Sevilla, Nonzero $\left|U_{e} 3\right|$ from Charged Lepton Corrections and the Atmospheric Neutrino Mixing Angle, JHEP 05 (2013) 073 [arXiv: 1302.0423] [INSPIRE].

[24] S.T. Petcov, Predicting the Values of the Leptonic CP-violation Phases, arXiv:1405.6006 [INSPIRE].

[25] M. Fukugita, M. Tanimoto and T. Yanagida, Atmospheric neutrino oscillation and a phenomenological lepton mass matrix, Phys. Rev. D 57 (1998) 4429 [hep-ph/9709388] [INSPIRE].

[26] V.D. Barger, S. Pakvasa, T.J. Weiler and K. Whisnant, Bimaximal mixing of three neutrinos, Phys. Lett. B 437 (1998) 107 [hep-ph/9806387] [INSPIRE].

[27] S. Davidson and S.F. King, Bimaximal neutrino mixing in the MSSM with a single right-handed neutrino, Phys. Lett. B 445 (1998) 191 [hep-ph/9808296] [INSPIRE].

[28] A. Datta, F.-S. Ling and P. Ramond, Correlated hierarchy, Dirac masses and large mixing angles, Nucl. Phys. B 671 (2003) 383 [hep-ph/0306002] [INSPIRE].

[29] L.L. Everett and A.J. Stuart, Icosahedral (A(5)) Family Symmetry and the Golden Ratio Prediction for Solar Neutrino Mixing, Phys. Rev. D 79 (2009) 085005 [arXiv:0812.1057] [INSPIRE].

[30] F. Feruglio and A. Paris, The Golden Ratio Prediction for the Solar Angle from a Natural Model with A5 Flavour Symmetry, JHEP 03 (2011) 101 [arXiv:1101.0393] [INSPIRE].

[31] C.S. Lam, Group Theory and Dynamics of Neutrino Mixing, Phys. Rev. D 83 (2011) 113002 [arXiv: 1104.0055] [INSPIRE].

[32] R. de Adelhart Toorop, F. Feruglio and C. Hagedorn, Finite Modular Groups and Lepton Mixing, Nucl. Phys. B 858 (2012) 437 [arXiv:1112.1340] [InSPIRE].

[33] W. Rodejohann, Unified Parametrization for Quark and Lepton Mixing Angles, Phys. Lett. B 671 (2009) 267 [arXiv:0810.5239] [INSPIRE].

[34] A. Adulpravitchai, A. Blum and W. Rodejohann, Golden Ratio Prediction for Solar Neutrino Mixing, New J. Phys. 11 (2009) 063026 [arXiv:0903.0531] [inSPIRE].

[35] C.H. Albright, A. Dueck and W. Rodejohann, Possible Alternatives to Tri-bimaximal Mixing, Eur. Phys. J. C 70 (2010) 1099 [arXiv: 1004.2798] [InSPIRE].

[36] S.T. Petcov and M. Piai, The LMA MSW solution of the solar neutrino problem, inverted neutrino mass hierarchy and reactor neutrino experiments, Phys. Lett. B 533 (2002) 94 [hep-ph/0112074] [INSPIRE].

[37] X. Qian et al., Mass Hierarchy Resolution in Reactor Anti-neutrino Experiments: Parameter Degeneracies and Detector Energy Response, Phys. Rev. D 87 (2013) 033005 [arXiv:1208.1551] [INSPIRE].

[38] S.-F. Ge, K. Hagiwara, N. Okamura and Y. Takaesu, Determination of mass hierarchy with medium baseline reactor neutrino experiments, JHEP 05 (2013) 131 [arXiv:1210.8141] [INSPIRE]. 
[39] Y.-F. Li, J. Cao, Y. Wang and L. Zhan, Unambiguous Determination of the Neutrino Mass Hierarchy Using Reactor Neutrinos, Phys. Rev. D 88 (2013) 013008 [arXiv:1303.6733] [INSPIRE].

[40] W. Wang, The neutrino mass hierarchy: How will we determine it?, talk presented at Invisibles '13, Lumley Castle Durham U.K. (2013).

[41] J. Park, Recent results from RENO, AIP Conf. Proc. 1604 (2014) 421 [InSPIRE].

[42] J. Park, Study of Neutrino Mass Hierarchy with RENO-50, PoS(Neutel 2013) 076.

[43] LBNE collaboration, C. Adams et al., The Long-Baseline Neutrino Experiment: Exploring Fundamental Symmetries of the Universe, arXiv:1307.7335 [INSPIRE].

[44] LAGUNA-LBNO collaboration, S.K. Agarwalla et al., The mass-hierarchy and CP-violation discovery reach of the LBNO long-baseline neutrino experiment, JHEP 05 (2014) 094 [arXiv: 1312.6520] [INSPIRE].

[45] P. Ballett, S.F. King, C. Luhn, S. Pascoli and M.A. Schmidt, Precision measurements of $\theta 12$ for testing models of discrete leptonic flavour symmetries, arXiv:1406.0308 [INSPIRE].

[46] S. Gollu, K.N. Deepthi and R. Mohanta, Charged lepton correction to tri-bimaximal lepton mixing and its implications to neutrino phenomenology, Mod. Phys. Lett. A 28 (2013) 1350131 [arXiv: 1303.3393] [INSPIRE].

[47] M. Sruthilaya, C. Soumya, K.N. Deepthi and R. Mohanta, Predicting Leptonic CP phase by considering deviations in charged lepton and neutrino sectors, arXiv:1408.4392 [INSPIRE].

[48] I. Girardi, S.T. Petcov and A.V. Titov, Determining the Dirac CP-violation Phase in the Neutrino Mixing Matrix from Sum Rules, arXiv:1410.8056 [INSPIRE].

[49] Particle Data Group collaboration, K.A. Olive et al., Review of Particle Physics, Chin. Phys. C 38 (2014) 090001 [INSPIRE].

[50] P. Coloma, A. Donini, E. Fernandez-Martinez and P. Hernández, Precision on leptonic mixing parameters at future neutrino oscillation experiments, JHEP 06 (2012) 073 [arXiv: 1203.5651] [INSPIRE].

[51] Intensity Frontier Neutrino Working Group collaboration, A. de Gouvêa et al., Working Group Report: Neutrinos, arXiv:1310.4340 [INSPIRE].

[52] R.M. Fonseca and W. Grimus, Classification of lepton mixing matrices from finite residual symmetries, JHEP 09 (2014) 033 [arXiv: 1405.3678] [INSPIRE].

[53] A. Blum, C. Hagedorn and M. Lindner, Fermion Masses and Mixings from Dihedral Flavor Symmetries with Preserved Subgroups, Phys. Rev. D 77 (2008) 076004 [arXiv:0709.3450] [INSPIRE].

[54] F. Capozzi, E. Lisi and A. Marrone, Neutrino mass hierarchy and electron neutrino oscillation parameters with one hundred thousand reactor events, Phys. Rev. D 89 (2014) 013001 [arXiv: 1309.1638] [INSPIRE].

[55] M. Grassi, J. Evslin, E. Ciuffoli and X. Zhang, Showering Cosmogenic Muons in A Large Liquid Scintillator, JHEP 09 (2014) 049 [arXiv:1401.7796] [INSPIRE].

[56] A. Stahl et al., Expression of Interest for a very long baseline neutrino oscillation experiment (LBNO), CERN-SPSC-2012-021 (2012).

[57] A. Longhin, Optimization of neutrino beams for underground sites in Europe, arXiv: 1206.4294 [INSPIRE]. 
[58] P. Huber, M. Lindner and W. Winter, Simulation of long-baseline neutrino oscillation experiments with GLoBES (General Long Baseline Experiment Simulator), Comput. Phys. Commun. 167 (2005) 195 [hep-ph/0407333] [INSPIRE].

[59] P. Huber, J. Kopp, M. Lindner, M. Rolinec and W. Winter, New features in the simulation of neutrino oscillation experiments with GLoBES 3.0: General Long Baseline Experiment Simulator, Comput. Phys. Commun. 177 (2007) 432 [hep-ph/0701187] [InSPIRE].

[60] S.F. King, C. Luhn and A.J. Stuart, A Grand Delta(96) x SU(5) Flavour Model, Nucl. Phys. B 867 (2013) 203 [arXiv: 1207.5741] [INSPIRE].

[61] M.A. Schmidt and A.Y. Smirnov, Quark Lepton Complementarity and Renormalization Group Effects, Phys. Rev. D 74 (2006) 113003 [hep-ph/0607232] [INSPIRE].

[62] S. Boudjemaa and S.F. King, Deviations from Tri-bimaximal Mixing: Charged Lepton Corrections and Renormalization Group Running, Phys. Rev. D 79 (2009) 033001 [arXiv: 0808.2782] [INSPIRE].

[63] S. Antusch, J. Kersten, M. Lindner and M. Ratz, Running neutrino masses, mixings and CP phases: Analytical results and phenomenological consequences, Nucl. Phys. B 674 (2003) 401 [hep-ph/0305273] [INSPIRE]. 